

\title{
Model of macroeconomic evolution in stable regionally dependent economic fields
}

\author{
M. Ausloos ${ }^{1}$, P. Clippe ${ }^{2}$ and A. Pekalski ${ }^{3}$ \\ ${ }^{1}$ GRASP and SUPRATECS, B5, Sart Tilman, B-4000 Liège, Belgium \\ 2 GRASP, B5, Université de Liège, B-4000 Liège, Belgium, \\ ${ }^{3}$ Institute of Theoretical Physics, University of Wroclaw, pl. Maxa Borna 9, PL-50-204 Wroclaw, Poland
}

(March 25, 2021)

We develop a model for the evolution of economic entities within a geographical type of framework. On a square symmetry lattice made of three (economic) regions, firms, described by a scalar fitness, are allowed to move, adapt, merge or create spin-offs under predetermined rules, in a space and time dependent economic environment. We only consider here one timely variation of the "external economic field condition". For the firm fitness evolution we take into account a constraint such that the disappearance of a firm modifies the fitness of nearest neighboring ones, as in Bak-Sneppen population fitness evolution model. The concentration of firms, the averaged fitness, the regional distribution of firms, and fitness for different time moments, the number of collapsed, merged and new firms as a function of time have been recorded and are discussed. Also the asymptotic values of the number of firms present in the three regions together with their average fitness, as well as the number of respective births and collapses in the three regions are examined. It appears that a sort of critical selection pressure exists. A power law dependence, signature of self-critical organization is seen in the birth and collapse asymptotic values for a high selection pressure only. A lack of self-organization is also seen at region borders.

PACS numbers: 789.65.Gh, 05.10.Ln, 89.75.-k, 07.05.Tp, 05.65.+b

\section{INTRODUCTION}

The present contribution extends our earlier paper, $[1,2]$. World economic conditions evolved and are quite varied on different time and space scales. Basic questions are whether the consequences of political conditions have predictable effects, or not and whether annoying situations can be avoided. The questions pertain to macroeconomic themes, not so often touched upon in econophysics (see exceptions in [3-5]).

The case of the Berlin wall fall followed by Eastern Europe and Central Asia market openings to a so called liberal economy is a intriguing event. From an econophysicist point of view, the event(s) can be considered

\footnotetext{
${ }^{1}$ electronic address: marcel.ausloos@ulg.ac.be

${ }^{2}$ electronic address: P.Clippeulg.ac.be

${ }^{3}$ electronic address: apekalift.uni.wroc.pl
}

as an increase in "physical volume, or available space" as well as in a modification of the external fields [1]. In the latter reference we have discussed whether one can describe, within a simple model, the concentration of enterprises and their so called "fitness" as a function of time and space, under varying in time and space economic field conditions. We have observed a non-trivial behavior with cycle features; in our opinion more pronounced that those discussed by Aoki, Kalecki, Freeman and others [5-8]. The model however seemed to be too optimistic in not allowing enough bankruptcies, collapses or mere disappearance of firms in the process, in particular with respect to the so called economic selection pressure $[1,2]$. Moreover we neglected the fact that a firm disappearance could locally modify the probability of survival of neighboring ones. Indeed an immense political worry concerns the effect of degradation of an economy through avalanche-like processes.

In practice, adaptation to a dynamic environment contains a trade-off: it is more difficult to adapt in the next (time) step as shown e.g. also on financial time series by Yamasaki et al. [9]. It is also expected that the adaptive behaviors of "populations" differ depending whether a Darwinian or Lamarckian scheme is implied. A large discussion exists whether such schemes hold or not, and how in economy, e.g. see [10-17] for references and a recent review. Alas, the definition of Lamarckism (or Darwinism) is not totally agreed upon in economy circles.

Therefore we present and discuss here below a more elaborate model than in Ausloos et al. [1,2], i.e. in order to describe changing basic economic conditions and implement more realistic Darwinian-like adaptation rules, as discussed in economy circles [10-17] - in space and time. In essence we introduce a Darwin-Bak-Sneppenlike constraint [18] in our previous macro-economy $[1,2]$ microscopic physics-like model, i.e. the choice of the initial firm or company depends on its relative fitness with respect to the external field as in [2]; the chosen firm is the less adapted one, instead of being chosen at random [1]. The fitness of neighboring ones evolve according to some a priori defined dynamics.

The notion of economic external field is also important. Nelson and Winter [17] write that "at any time, firms in an industry can be viewed as operating with a set of techniques and decision rules (routines) keyed to conditions external to the firm" ... "and to various internal state conditions". Surely self-organization and external 
(field) conditions exist at the same time, and are hard to separate $[19,20]$. However there are also many different conditions affecting many characteristic variables of a company or industry state. Thus searching for as simple as possible description and consequences of such external though often qualitatively only apparent constraint, to be contrasted to self-organization is of interest. As usual in physics when intending extreme simplification this field should be applied toward or coupled to some variable such that some function be optimized [21], like the free energy in thermodynamics. In macro and micro-economy the field can be understood as of political origin, but also of more physical origin like the weather. In the latter case, see the weather influence on the energy prices [22]. As example of the former one can think about deregulation, or wage and benefit taxation influences upon localization and production or market approach by a company.

Along this line, the most extreme simplification leads to represent a company by a variable having the same number of characteristic symmetry group elements as the field. Thus a scalar, called the fitness of a company is introduced $[1,2]$. One can consider that the fitness is the price of stocks and the external field can be in some sense the value of the S\&P500 to which the price is compared. A buy/sell strategy will depend on the difference between the two values. A too large difference can suggest bankruptcy and disappearance of a company, or the 'need" for a modification of the business plan. The notion of fitness of a company is related to such a book value. The fitness might also represent the number found on gross sales or benefit lines.... The field notion has to be subsequently adapted.

Any such system will be evolutionary if it has a fourth ingredients: beside the set of degrees of freedom, each ranked by a quality fitness criterion, and a mechanism for introducing mutations to interject diversity into the productive process, the system must be governed by a selection process based on the fitness ranking $[6,23,24]$ as in biology [25].

Thus we consider a system composed of firms located at the nodes of a locally square symmetry lattice of $L_{x}, L_{y}$ size dimensions. As in Ausloos et al. [1] the system is divided into three parts, $k=$ I, II and III, by vertical boundaries at $L_{x k}$, i.e. lines along the $y$ axis at $L_{x 1}, L_{x 2}$. There is no periodic boundary conditions. At the beginning there are $N(t=0)$ firms, located only in the first (I) region. One lattice site may be occupied by only one firm. Apart from its location on the lattice a firm $i$ is characterized by its scalar fitness, $f_{i}$, taken from a uniform distribution, $\in(0,1)$. There is no other factor distinguishing the firms, hence we may consider them to belong to only one group of industrial companies, i.e. representing a particular industrial branch. Initially the fitness values are attributed to firms in a random way.

Any firm may change its position [20] to a vacant nearest neighboring site chosen at random and may either merge with another firm (with a given probability, - ac- cording to its business plan) or create a new one. In a region, a firm is under an external field $F \in(0,1)$, which characterizes the local economic conditions. The local political system may be more or less demanding,- more or less high taxes, salary rules, worker age (and other) conditions, ...; this is taken into account through the parameter, sel, - corresponding to selection in biological systems [25]. In our model it is also a scaling parameter for the field and the fitness, favoring or not the possible disappearance of a company unfit to its environment, the field [26-28].

The field $F$ may (and will) differ from region to region and may also change in time, measured in Monte Carlo Steps (MCS). In the following we will only consider one drastic field change and keep stable (or static) all environmental (economic field) conditions for evaluating its effect on the population of entities evolution.

As in Ausloos et al. [1] after some time the barrier separating the region I from regions II and III is open and the diffusing firms may enter the latter regions. At that time the external field also changes in the first region and some values are given to the fields in the regions II and III.

Before any activity is taken for the firm (moving, merging...) its condition is checked by comparing its fitness with the field $F$. If the two values differ strongly, the firm may collapse and be removed from the system (see the algorithm below).

The Bak-Sneppen condition, i.e. a modification of the fitness of the eliminated firm nearest neighbors, is implemented through distributing new (uncorrelated in space and time) random fitness to these (at most 4) neighbors. This corresponds to e.g. simulating the effect a bankruptcy may have on other firms connected to the one which went down. Obviously the fitness neighbors can increase or decrease. Some firms may profit or regret the disappearance of a competitor in the local market.

Firms will also be systematically moving on the lattice, looking for partners in view of merging or producing spinoffs. We will take into account the number of contacts in the company network (neighbors on a lattice in our case) for describing the firm evolution. It is empirically known that the replacement of some contacts is needed for innovation [26-29].

The (new) firm(s) fitness evolution rule is slightly different from that in Ausloos et al. [1], but still takes into account the fitness of parents.

The following quantities are recorded: concentration of the firms in the three regions and the averaged fitness in the three regions both as a function of time, the spatial distribution of firm concentration and fitness for different time moments, the number of collapsed, merged and new firms as a function of time, finally the asymptotic, i.e. when a stationary state is reached, values of the number of firms present in the three regions together with their average fitness, as well as the number of respective birth and collapses.

In section 2 , we outline the simulation technique used 
for implementing the model. We present a few results in Sect. 3, and end with a conclusion in Sect.4.

\section{MODEL}

The algorithm we implemented is the following one:

1. from a number of firms $N(t)$ at a given time $t$ a firm $j$ is randomly picked

2. its survival probability is calculated from the condition

$$
p_{j}=\exp \left(-s e l\left|F-f_{j}\right|\right)
$$

and compared to a random number $r$ taken from a uniform distribution $\in(0,1)$. If $r>p_{j}$, the firm collapses, i.e. it is removed from the system; the nearest neighbors of the eliminated firm receive new random numbers (taken from a uniform distribution) for their fitness. The algorithm then goes back to 1 ,

3. if $r<p_{j}$, we try to move the firm to a nearest neighborhood (NN). A random number, $r_{1}$, is generated from a uniform distribution and if it is smaller than 0.25 then we check whether the "Northern NN" is an empty site, and we move the firm there, if possible; if $r_{1}$ is between 0.25 and 0.50 then we look into the Western NN, etc. If the displacement trial is not successful the algorithm goes back to 1 and search for a new firm;

4. next we look for a partner in the nearest neighborhood of the displaced firm new position. If there is a firm in the NN,

5. with a probability $(b=) 0.01$ the neighbor, say $i$, merges with the old firm $j$, which changes its fitness to

$$
f_{j}=0.5\left(f_{i}+f_{j}+\left(0.5-r_{2}\right)\left|f_{i}-f_{j}\right|\right)
$$

where $r_{2}$ is a random number in the range $(0,1)$. The firm $i$ then disappears from the system;

6. otherwise with a probability $(1-b=) 0.99$ the firms $i$ and $j$ produce a new firm $k$, a spin - of $f$. The $k$ firm is randomly positioned in the Moore neighborhood of the $j$ firm on an empty site if any exists. The procedure for finding an empty place is similar to the one used when looking for an empty site to move the firm, except that on a square lattice the Moore neighborhood consists of 8 sites - NW, N, NE, W, E, SW, S and SE; whence if the random number $r_{3}$ is smaller than 0.125 we check the site NW, if it is larger than 0.125 but smaller than 0.250 we check the $\mathrm{N}$ site, etc. The new firm receives its fitness depending on that of both parents (which remain in the system) as

$$
f_{k}=0.5\left(f_{i}+f_{j}+\left(0.5-r_{4}\right)\left|f_{i}-f_{j}\right|\right)
$$

where $r_{3}$ and $r_{4}$ are random numbers in the range $(0,1)$.

7. When $N(t)$ firms were picked through (1), one MCS is said completed.

Typical values taken for the discussed simulations below were : $L_{x 1}=50, L_{x 2}=100, L_{y}=201, c(0) \simeq 0.8$, corresponding to a number of firms $N(0)=8040$.

The parameters of the model are: sel, selection pressure; $t_{\text {change }}$, time after which the barrier at $L_{x 1}$ is open and the field changed in I; the values of the fields. At the beginning the field in region $I$ has the value $F_{I}=$ 0.5 , which is "optimal"; the values in the regions II and III are irrelevant before the opening of the $L_{x 1}$ barrier, since there are no firms there. After the change, at time $t_{\text {change }}=100 \mathrm{MCS}$, the values were arbitrarily taken to be: $F_{I}=0.3, F_{I I}=0.5, F_{I I I}=0.6$, which means that the conditions in the region I deteriorated and the best situation is in region II. The conditions in region III are also imposed to be better than those in region I. These values remain unchanged till the end of the simulations.

Although the presented curves were obtained each from a single simulation, we have checked that a different initial distribution of the firms (for the same initial concentration, external field and selection) leads to a very similar, even quantitatively, set of situations. We are not interested here in the transient regime before the first 100 MCS. The simulations were carried out till a stationary state was reached, when each investigated quantity mildly oscillated around a rather stable average value. This happened quite soon (several hundreds of MCS) for low selection, but we had to run till 20000 MCS for the highest selection $\mathrm{sel}=1.7$. For a sel as "high" as 1.8 , we found that no firm survived after the initial 800 MCS in the whole system.

\section{RESULTS AND COMMENTS}

There are several ways of presenting pertinent results. In the following we stress cases demonstrating the pertinence of the model in view of economic qualitative observations. The scales are chosen in order to allow for better visually comparing cases and emphasizing differences in behavior.

Recall that from time $t_{\text {change }}=100 \mathrm{MCS}$, the field values are kept to be $F_{I}=0.3, F_{I I}=0.5$, and $F_{I I I}=$ 0.6 respectively. 


\section{A. Time dependent and regional values}

In Fig. 1 (a-b) the number of new firms appearing in each region is shown as a function of (MC) time, (a) in the case of a rather low sel, i.e. $=0.7$ and in the case (b) of a rather high sel $=1.7$. In Fig. $2(\mathrm{a}-\mathrm{b})$ the number of eliminated firms versus time, for the above two values of sel are shown. It is worth pointing here, the difference with respect to the case examined in ref. [1]. In the latter paper the long time stability (asymptotic value) was obtained through a sharp increase followed by an exponential decay toward a zero death (and also birth) evolution. In the present case, the number of born or eliminated firms increases smoothly and remains stable at a finite value. Recall that there are 8000 firms ("possible parents") at $t=0$. Observe the $x$-axis scale indicating the effect of selection pressure on first time invasion of regions II and III.

A minimum in the birth and death number is always observed at short time (after $100 \mathrm{MCS}$ ) in region I. Notice from Fig. 1(b) and Fig. 2(b) that a strong sel might quickly kill any evolution process : zero-birth and zero death. It is also clear from comparing Fig. 1 and Fig. 2 that there is a rapid increase in concentration in the newly opened regions as soon as the barrier at $L_{x 1}$ is removed. The death and birth processes are always quantitatively equivalent, indicating that a detailed balance equilibrium is intrinsically met in the model.

The number of firms present in the three regions vs. time (in MCS) is shown for several sel values, from 0.4 till 1.7 in Fig.3 (a-d). At very low sel the number of firms rather quickly reaches a rather high saturation value in each region, in fact approximately equal to the initial value in region I. At high sel, Fig. $3(\mathrm{c}-\mathrm{d})$, there is a marked drop in region I, while regions II and III are invaded much later than in Fig.3 (a); the saturation level has a lower value than the initial starting one in region I. The asymptotic number of firm values is seen to decrease with sel. The strongly marked oscillations might be considered as a signature of cycles [5,8,24]. At high sel values the effects are much enhanced. Also, regions II and III are invaded very slowly and late in time. Yet the fitness optimal value in each region is easily reached and remain stable in time (see Fig.4 below).

In Fig. 4(a-d) we show the average fitness $f$ in the three regions vs. MCS time for several values of the selection pressure, ranging from 0.4 to 1.7 . Recall that the values of the external field are respectively $0.3,0.5$ and 0.6 , and the wall at $L_{x 1}$ falls at $t=100$. For a low sel $(=0.4)$ small fluctuations are seen around the average (asymptotic) values which are close to 0.5 in all regions. Since the selection pressure is low, the firms are not strongly forced to being in perfect matching to the field value, whence large deviations from the regional average fitness can be enforced.

For higher sel, data is quite scattered, and indicate a strongly hard learning (adaptation) process, but the av- erage fitness in region $\mathrm{I}$ is near 0.3 , and that in region III close to 0.6 as expected. For high sel large fluctuations in the average $f$ value are visible as in Fig. 4(b), at rather short time. At very large sel the beginning of the process is rather noisy, but the average fitness stabilizes at a value expected from the imposed external field. We adopted scales for the figures in order to show qualitatively the decrease in the variance of the fitness distribution with time, for various selection pressures. The adaptation is clearly faster in region I and II, and the variance much reduced as soon as the expected average values are obtained.

Considering the physical diffusion process, we recorded the maximum distance reached by the right most firm as a function of time, and present it on a log-log scale for different sel values, from 0.3 till 1.8, in Fig. 5. Recall that due to the lattice topology, critical values are to be found at $x=50,100$ and 150. Studying Fig. 5, starting from high sel values, it appears that a plateau occurs (for sel $=1.8$ and 1.5) near $L_{x 2}=100$, also somewhat seen for sel $=1.1$. The flow into region II and III seems more continuous at small sel values. The small tails sticking upwards (above $x=50$ ) for $t>1000$ witness unsuccessful attempts to colonize regions II and III, suggesting that a sel-dependent nucleation-growth process exists in the model. Moreover, for high sel values it appears that the region near the border at $L_{x 1}=50$ is sometimes depleted., indicating a gain that high sel values might destroy the population at all. From Fig. 5 (and others not shown here) we can calculate the time necessary for the first firm to reach the region right boundary, i.e. respectively $L_{x 2}=100$ and $L_{x 3}=150$, as a function of the selection pressure (Fig. 6). The curves look like high order power laws (with exponents equal ca. 4 and 5 respectively), but more complicated functions might be tried.

\section{B. Asymptotic values}

It is of interest to observe beyond the transient regimes the equilibrium values. In Fig.7 (a) the normalized asymptotic values of the born firms in the three regions are shown as a function of sel. The "normalization" is made with respect to the total asymptotic number of firms in the region shown in Fig. 7(b). "Asymptotic" meaning here that we have averaged the last 500 entries for 10000 MCS runs. The same type of data is obtained quantitatively for the number of disappearing firms (not shown). A maximum occurs near $\mathrm{sel}=0.8$ with different analytic behaviors on both sides of this value. The increase goes like a stretched exponential as for diffusioncontrolled growing entities [30] at low sel $(<0.8)$, i.e. $s e l^{0.45} \exp (-0.5 / \sqrt{(s e l)})$, but the decay at high sel has been found to be markedly a power law $\operatorname{sel}^{-0.40}$, like for critical systems [31]. Recall that each region may contain at most 10050 entities, a number to be compared to the number of asymptotically existing firms, as displayed on 
Fig. 7 (b). For completeness, the asymptotic fitness in the three regions is shown vs. sel in Fig. 8. This indicates that only for large sel $(>0.8)$ has the asymptotic average fitness almost reached the expected one from the field conditions.

\section{Regional behaviors}

We show the short time dependence $(t \leq 800$ MCS) of the (average over a "vertical column") firm concentration vs. the column number " $x$ " for rather strong (1.3) and very strong (1.7) sel values in Fig. 9. A drop in the overall concentration is well seen in region I at short time, but the concentration recovers later on, as seen from previous paragraphs and figures. For sel $=1.8$ the selection pressure is too high to maintain any entity in region I after a few MCS.

The dependence of the firm (vertical) concentrations on position " $x$ " at different (longer) times is shown in Fig. 10 (a-c) for sel values just above the critical one, i.e. $\simeq 0.8$.

Interesting and unexpected features occur. At very low sel (not displayed) a saturation level is reached right from the beginning of the simulation, indicating "easy adaptation" everywhere. At high sel the third region is invaded $c a$. after 5000 MCS, while region II is invaded after 800 MCS. We emphasize the occurrence of large well marked "oscillations" in the local concentrations. Dips do occur, see Fig. 10 near the region borders, - likely due to the difficulty for firms to reach the most appropriate fitness together with their neighbors, due to the interregion field gradient.

Finally the (vertically averaged) fitness vs. position along the $x$-axis taken at three time steps and for two sel values on both sides of the critical one are shown in Fig. 11. Notice the different time(s) chosen for such snapshots. For medium sel $(=0.7)$, the scatter around the expected theoretical fitness imposed by the external field is not large but the average values nevertheless do not closely correspond to the expected ones from the imposed field, in all three regions, - the more so on the average, and asymptotically as seen above. At high sel $(=1.7)$, the data is more scattered but the expected values are better recognized as fulfilling the external field condition.

\section{CONCLUSION}

The dynamics of an economic firm population has been considered through a model considering entities characterized by a scalar number, diffusing on a regular lattice, merging, collapsing or creating spin-offs. The economic environment is described by a static regionally dependent field. The adaptation and evolution of the firms to the field condition have been mimicked by a Darwinian-like selection rule but with a fitness evolution equation for the newly appearing firms taking into account that of the parents. The firm fitness distribution is shown to have some best adaptation difficulty, as discussed by Yamasaki et al. [9]. We have found an unexpected and interesting feature: the local changes of the environment is leading to sharp variations, almost discontinuous ones, in the fitness and concentrations, in particular when the field gradient is strong. A lack of self-organization is thus seen at region borders. This is due to the fact that for an entity attempting an adaptation, the learning process is quite hill climbing as in NK models [32]. Indeed the evolution of an economy, in which the functioning of companies is interdependent and depends on external conditions, through natural selection and somewhat random mutation is similar to bioevolution on NK fitness landscapes as described by Kaufmann [32]. In these evolutionary economics models [28], economic agents randomly search for new technological design by trial-and-error and run the risk of ending up in sub-optimal solutions due to interdependencies between the elements in a complex system. As argued by Frenken [28] these models of random search are legitimate for reasons of modelling simplicity, but remain limited as these models ignore the fact that agents can apply heuristics." We totally agree. Indeed there is no mimicking nor endogeneous search for optimisation as in [26-28] in the present model, the algorithm being only geared toward reducing the distance between the company fitness and the external field value.

One might take into account in a more proper way the number of business contacts, the evolving firm neighbors : the development of a market or a business plan, or a macroeconomy depends on the available number of contacts (bonds between nodes on a network) whence to the creation and destruction of contacts. A large stock of relational capital (business contacts) usually increases the sold output of a representative firm. Other lattices or network structures should be usefully studied.

It would be of interest to consider specific (historical) cases, and connect the MC simulations time scales to real cases. The time scale of changing environments is an available parameter which could indicate how robust the model can be with respect to realistic adaptation and evolution. Some flexibility for defining time scales exist in the (i) $b$ and (ii) sel values: (i) The value of the spinoff creation probability might be too large for previous economies. It has been really shown in the line of the ACP model that for the best fitted company a small $b$ value leads to chaotic behavior while a large $b$ leads to a monopoly like situation [24]. (ii) A sel (space and time dependent) value could be found by examining macroeconomy data. A sort of critical selection pressure exists separating different birth-death regimes. This might be used as a time and field scale definition when counting bankruptcies in countries, including per industry type.

Contrary to the Bak-Sneppen model [18], the average fitness(es) or concentration(s) do not seem to appear as power laws (like those characterizing avalanches), except 
in the high selection pressure regime and for long (asymptotic) times. The power law dependence, which is a usual signature of self-critical organization is only seen for a high selection pressure in the asymptotic behavior of the birth-death number. Nevertheless from the set of results, as presented here above, we observe that in such a Darwinian evolving economic world: (i) there are relatively well marked effects due to the "selection pressure"; (ii) temporal field changes can imply a stable density distribution, as in Ausloos et al. [1]; (iii) a diffusion process together with a business plan, and the selection pressure smoothly leads to asymptotic equilibrium states with respect to birth and death processes; (iv) the fitness does not always reach the a priori externally imposed field value; (v) the role of the gradient (economic) field at borders might indicate either complex oscillations or chaotic behaviors in such regions.

We are aware that further improvements are needed. We are drastically caricaturing macro and micro economy field conditions, as well as the description of the "internal" interactions sequence(s). Surely a company, or a set of industries, should not be described by one scalar number $f_{i}$, but rather a vector (or matrix) model coupled to a (so called external) vector (or matrix) field should be examined. Moreover the birth and death process description through merging and spin off's could also be stochastic or evolutive. The spatial distribution in the distinct regions might be also on interest [29] as in biology [33].

\section{Acknowledgements}

MA thanks Masano Aoki, Michèle Sanglier, Mieko Tanaka-Yamawaki and Kazuko Yamazaki for stimulating discussions, references and comments.

[1] M. Ausloos, P. Clippe, A. Pȩkalski, Simple model for the dynamics of correlations in the evolution of economic entities under varying economic conditions, Physica A 324 (2003) 330.

[2] M. Ausloos, P. Clippe, A. Pȩkalski, Evolution of economic entities under heterogeneous political/environmental conditions within a Bak-Sneppen-like dynamics, Physica A 332 (2004) 394(physics/0309007).

[3] G.R. Richards, Reconciling econophysics with macroeconomic theory, Physica A 282 (2000) 325.

[4] M. Gligor, M. Ignat, A kinetic approach to some quasilinear laws of macroeconomics, Eur. Phys. J. B 30 (2002) 125.

[5] M. Aoki, A simple model of asymmetrical business cycles: Interactive dynamics of a large number of agents with discrete choices, Macroeconomic Dynamics 2 (1998) 427.

[6] M. Aoki, Y. Shirai, A New Look at the Diamond Search
Model: Stochastic Cycles and Equilibrium Selection in Search Equilibrium, Macroeconomic Dynamics 4 (2000) 487.

[7] M. Kalecki, A Macroeconomic Theory of the Business Cycle, Econometrica 3 (1935) 327; ibid., A Theory of the Business Cycle Review of Economic Studies 4 (1937) 77; ibid., Theory of Economic Dynamics: An essay on cyclical and long-run changes in capitalist economy, Monthly Review Press, New York, 1965.

[8] C. Freeman, Schumpeter's Business Cycles Revisited, in A. Heertje and M. Perlman (eds) Evolving Technology and Market Structures pp. 17-38 (1990)

[9] K. Yamasaki, K. Kitakaze, M. Sekiguchi, Adaptation under the Dynamic Environment and Application to Financial Time Series, IPSJ SIGNotes Intelligence and Complex Systems Abstract No.127 - 014

[10] A. Alchian, Uncertainty, Evolution, and Economic Theory, J. Pol. Econ. 58 (1950) 211.

[11] J. Hirshleifer, Economics from a Biological Viewpoint, Journal of Law and Economics 20 (1977) 1.

[12] K. Boulding, What is Evolutionary Economics?, J. Evol. Econ. 1 (1991) 9.

[13] N. Eldredge, Evolution in the Market Place, Structural Change and Economic Dynamics 8 (1997) 385.

[14] M. Kelm, Schumpeter's Theory of Economic Evolution: A Darwinian Interpretation, Evolutionary Economics 7 (1997) 97.

[15] G.M. Hodgson, "Darwinism in economics: from analogy to ontology, J. Evol. Econ. 12 (2002) 259.

[16] J. van den Bergh, J. Gowdy, The Microfoundations of Macroeconomics: An Evolutionary Perspective, Cambridge Journal of Economics 27 (2003) 65.

[17] R.R. Nelson, S. G. Winter, An Evolutionary Theory Of Economic Change, Cambridge, Belknap Press, 1982.

[18] P. Bak, K. Sneppen, Punctuated equilibrium and criticality in a simple model of evolution, Phys. Rev. Lett. 71 (1993) 4083.

[19] J. Foster, The Self-Organization Approach in Economics, Structural Change and Economic Dynamics 8 (1997) 427.

[20] G. Silverberg, G. Dosi, L. Orsenigo, Innovation, Diversity and Diffusion: A Self-Organization Model, The Economic Journal 98 (1998) 1032.

[21] P. Dutta, R. Radner, Profit Maximization and the Market Selection Hypothesis, Review of Economic Studies 66 (1999) 769 .

[22] R. Weron, B. Przybyłowicz, Hurst analysis of electricity price dynamics, Physica A 283 (2000) 462.

[23] M. Aoki, Assymetrical Cycles and Equilibrium Selection in Finitary Evolutionary Economic Models, in Cycles, Growth, and Structural Changes, L. Punzo Ed., (Routledge, London, 2001) Ch. 8

[24] J. Miśkiewicz, M. Ausloos, Logistic map approach to economic cycles, Physica A, submitted.

[25] A. Pȩkalski, K. Sznajd-Weron, Population dynamics with and without selection, Phys. Rev. E 63 (2001) 31903.

[26] S.A. Kauffman, J. Lobo, W.G. Macready, Optimal search on a technology landscape, J. Econom. Behav. Organiz. 43 (2000) 141.

[27] P. Auerswald, S. Kauffman, J. Lobo, K. Shell, "The Production Recipes Approach to Modeling Technological In- 
novation: An Application to Learning By Doing," Cornell University, CAE Working Paper 98-10, 1998.

[28] K. Frenken, Innovation rules: the NK-model as a framework for memetics, unpublished (2001); K. Frenken, L. Marengo, M. Valente, Interdependencies, neardecomposability and adaptation, pp. 145-165 in: Brenner, T. (ed.), Computational Techniques for Modelling Learning in Economics ( Kluwer, Boston, 1999); http : //www - ceel.gelso.unitn.it/papers/papero99_03.pdf

[29] M. Aoki, Cluster Size Distribution of Economics Agents of Many Types in a Market, J. Math. Anal. Appl. 249 (2000) 32 .

[30] D. Crespo, T. Pradell, M. T. Clavaguera-Mora, N. Clavaguera, Microstructural evaluation of primary crystallization with diffusion-controlled grain growth, Phys. Rev. B 55 (1997) 3435.

[31] H. E. Stanley, Scaling, universality, and renormalization: Three pillars of modern critical phenomena, Rev. Mod. Phys. 71 (1999) S358.

[32] S.A. Kauffman, S. Levin, Towards a general theory of adaptive walks on rugged landscapes, J. Theor. Biol. 128 (1987) 11.

[33] M. Ausloos, I. Mroz, A. Pȩkalski, N. Vandewalle, Lattice gas model of gradual evolution, Physica A 248 (1998) 155 .

\section{FIGURE CAPTIONS}

Fig. 1 - Number of new firms born in region I, II, III ( ,$+ \mathrm{x}$, dotted square, respectively) as a function of time, (a) sel $=0.7 ;$ (b) sel $=1.7$

Fig. 2 - Number of eliminated firms in the three regions versus time, for (a) sel $=0.7$; (b) sel $=1.7$

Fig. 3 - Number of firms in the three regions vs. time (in MCS) for (a) sel $=0.4$; (b) sel $=0.9$; (c) sel $=1.3$; (d) sel $=1.7$

Fig. 4 - Average fitness in the three regions I, II, III (,$+ \mathrm{x}$, dotted square, respectively) vs. MCS time for several values of the selection pressure $(\mathrm{a})=0.4 ;(\mathrm{b})=$ $0.9 ;(\mathrm{c})=1.3 ;(\mathrm{d})=1.7$

Fig. 5-Maximum distance reached by the right most firm as a function of time, on a log-log scale for different sel values

Fig. 6 - Shortest time necessary for a firm to reach the region II (100) or III (150) right boundary, i.e. $L_{x 1}=100$, $L_{x 2}=150$, as a function of the selection pressure ; curves indicate the best power law fit, i.e. ca. 4 (top) and 5 (bottom) respectively
Fig. 7 - (a) Normalized asymptotic values values of the born firms in the three regions as a function of various sel; "Normalization" is with respect to (b) total asymptotic number of firms in the respective region

Fig. 8. - Asymptotic fitness in regions I, II and III vs. sel ; recall that the field values are imposed to be $0.3,05$. and 0.6 respectively

Fig. 9 - Short time dependence of the firm concentration in regions I, II, III vs. spatial coordinate " $x$ " for sel values $(\mathrm{a})=1.3,(\mathrm{~b})=1.7$ and at different time moments - 10,20,50 and 800 MCS.

Fig. 10 - Dependence of the firm concentrations on position " $x "$ at different times for $s e l=(\mathrm{d}) 1.1$; (e) 1.5 ; (f) 1.7

Fig. 11 - Vertically averaged fitness vs. position along the $x$-axis at three time steps and for two sel values, i.e. (a) 0.7 and (b) 1.7 


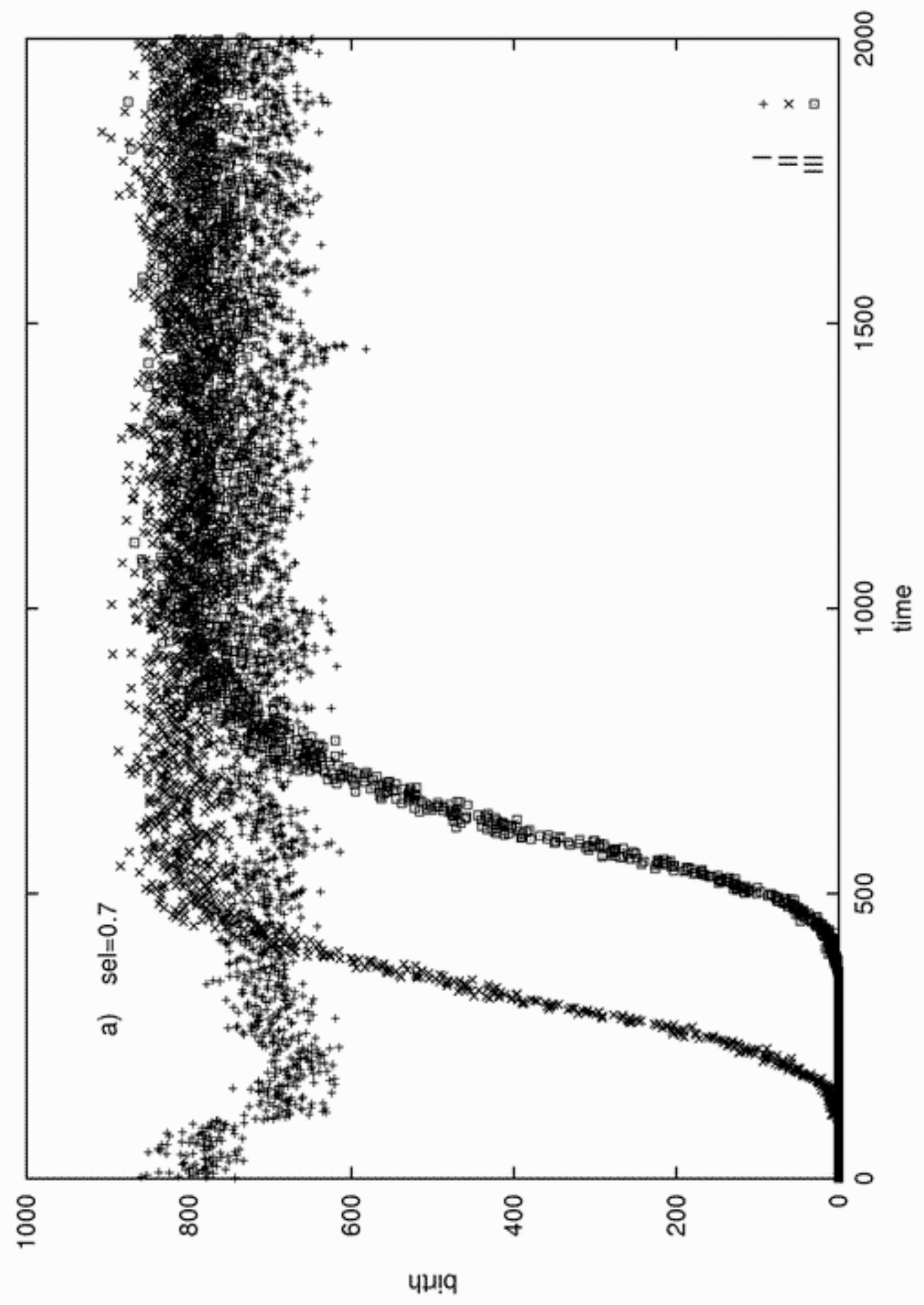




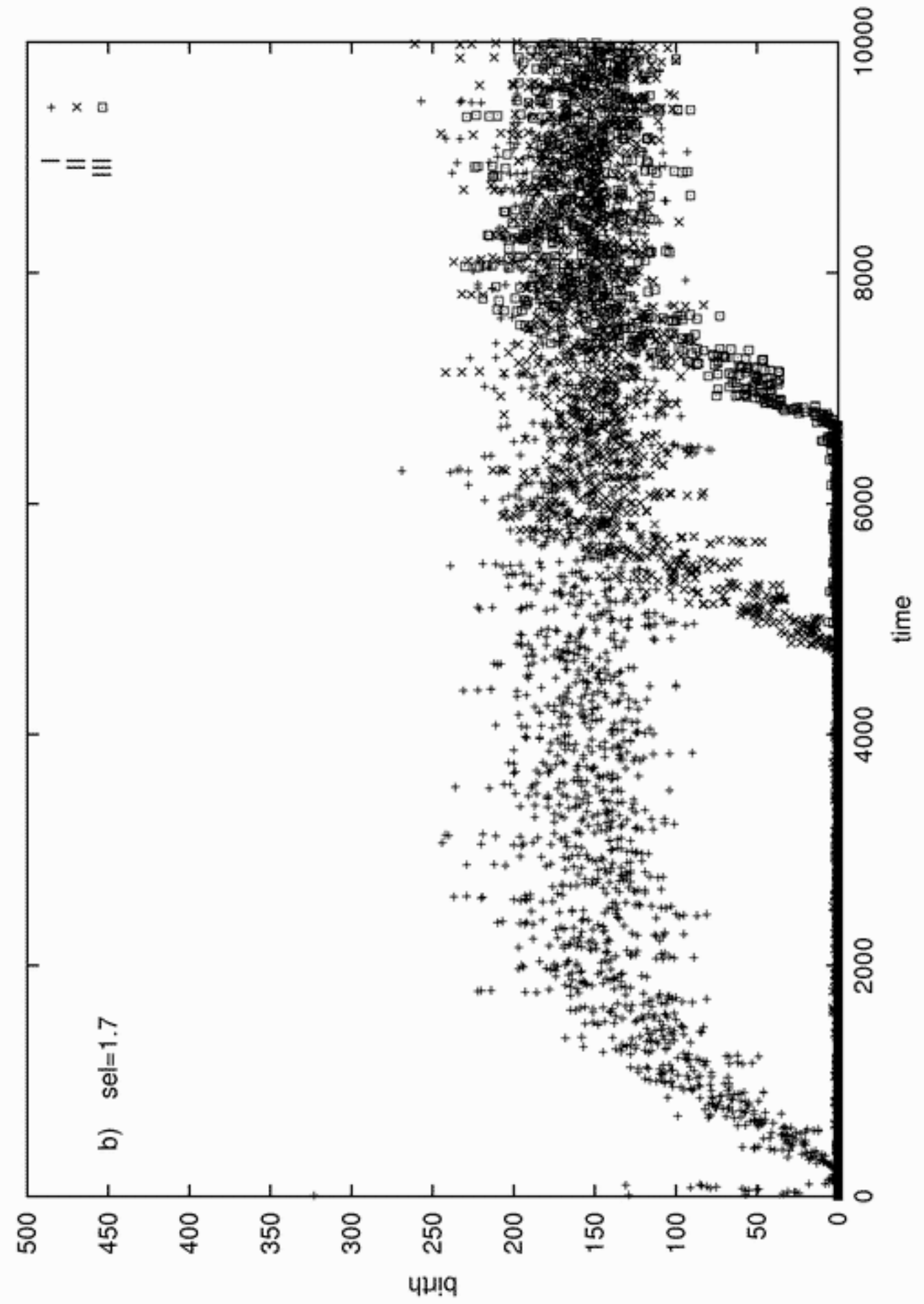




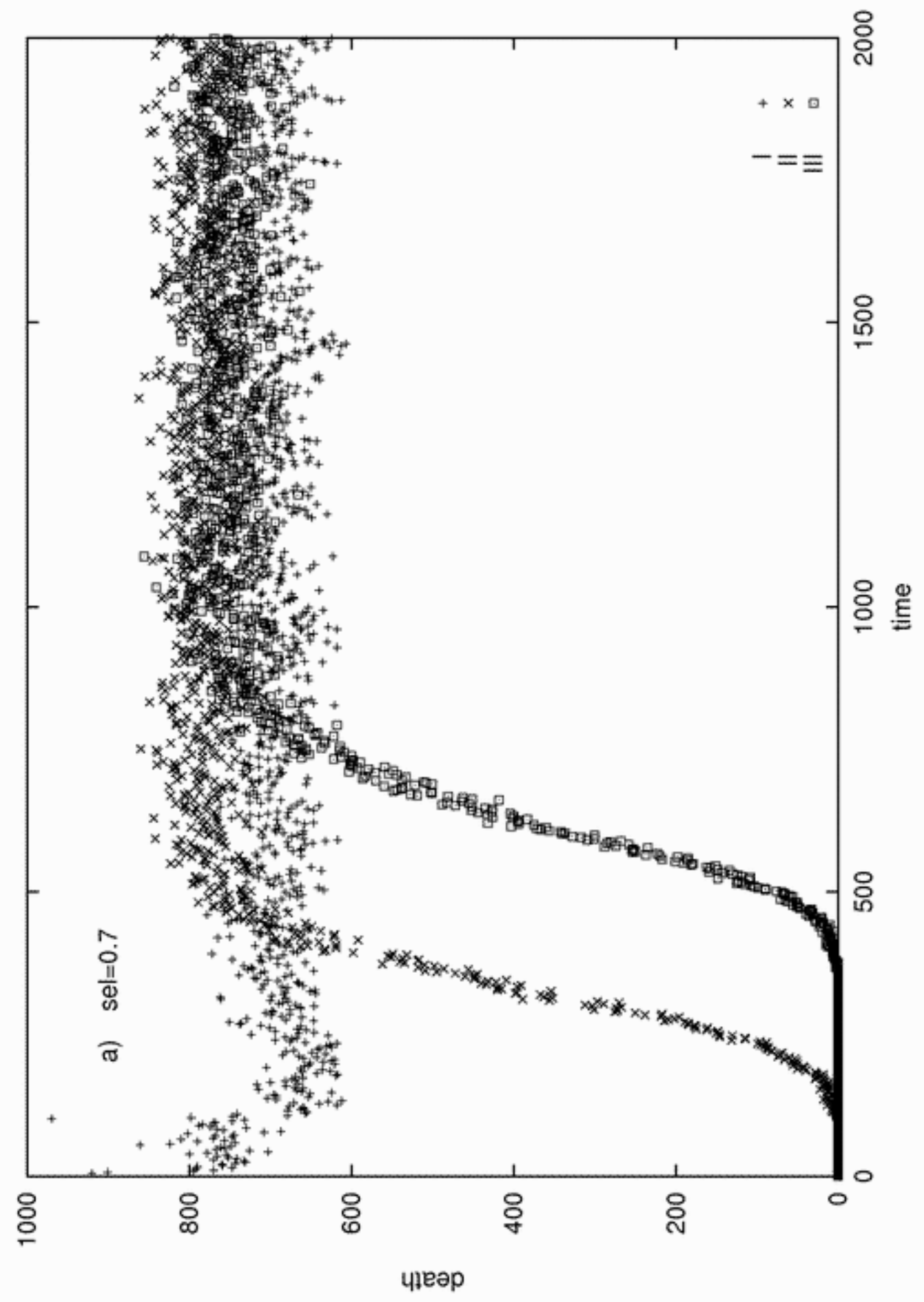




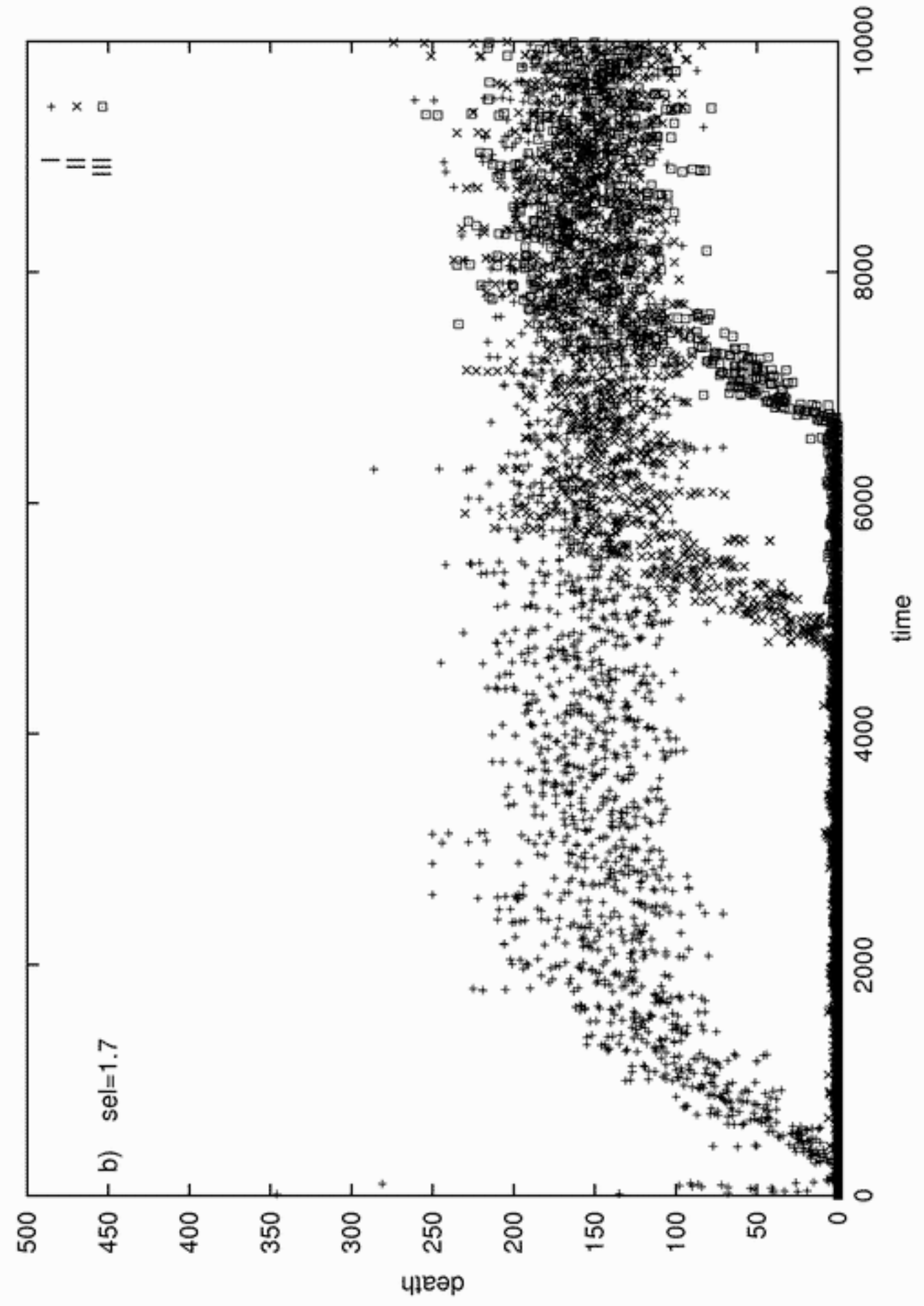




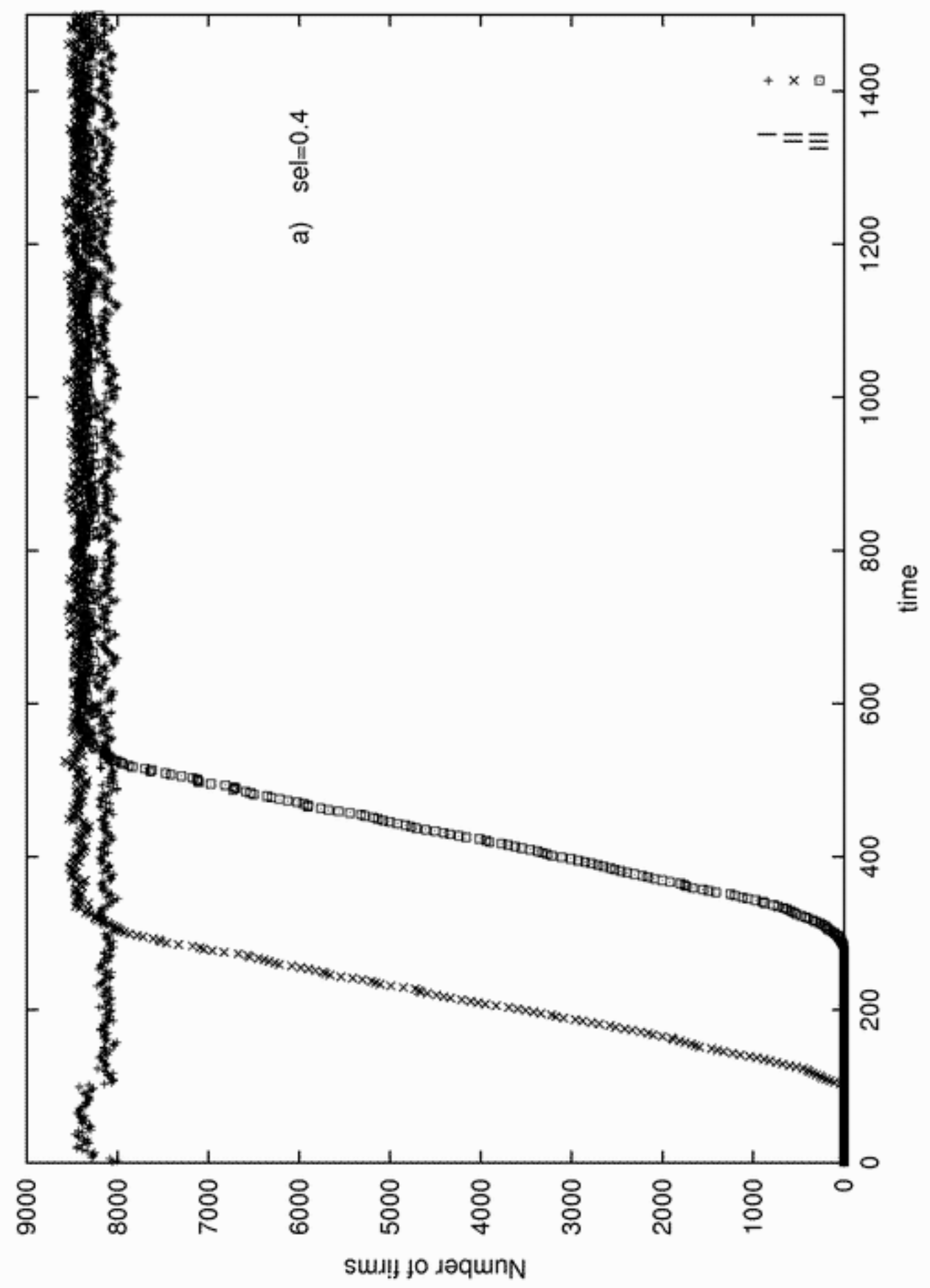




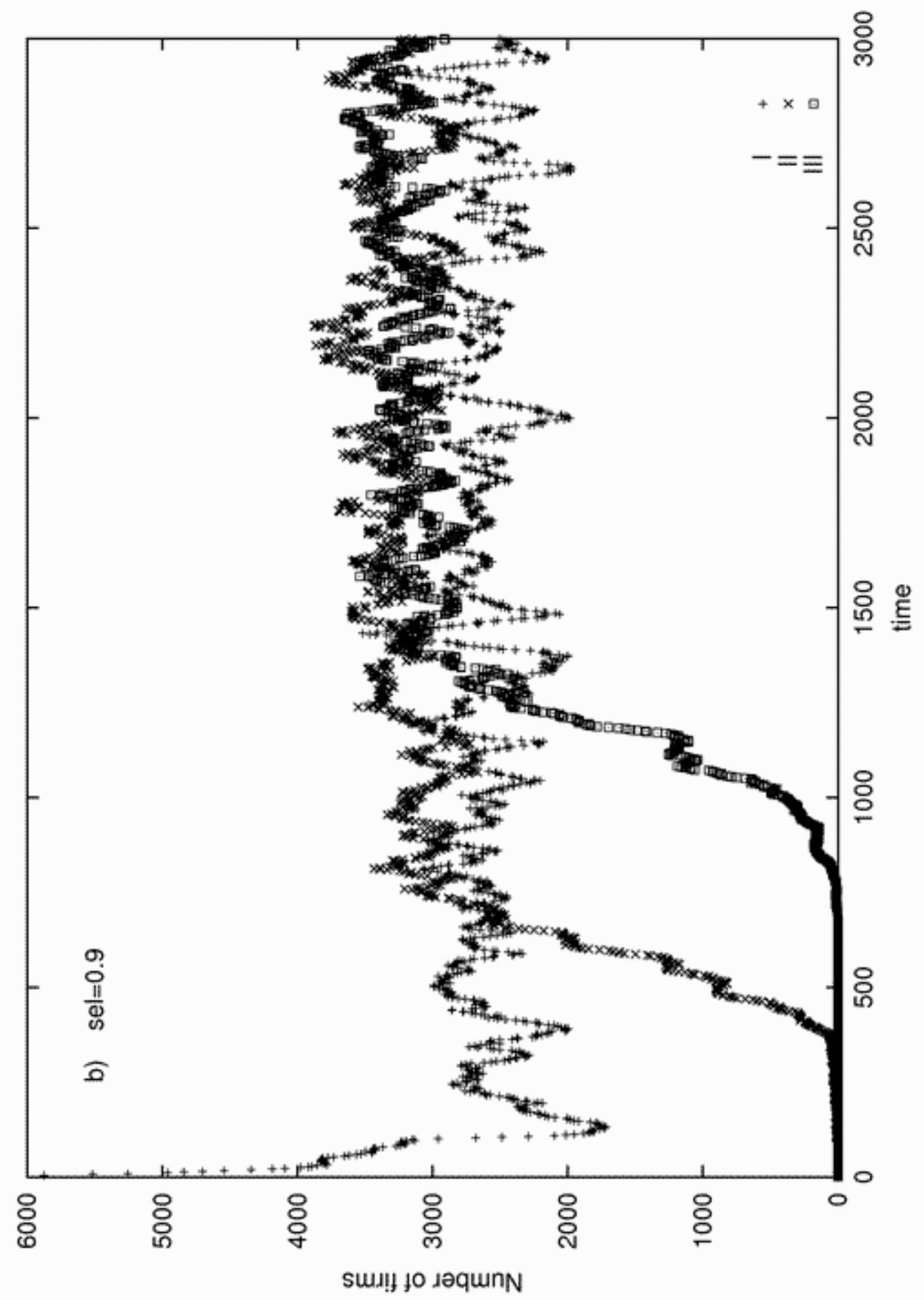




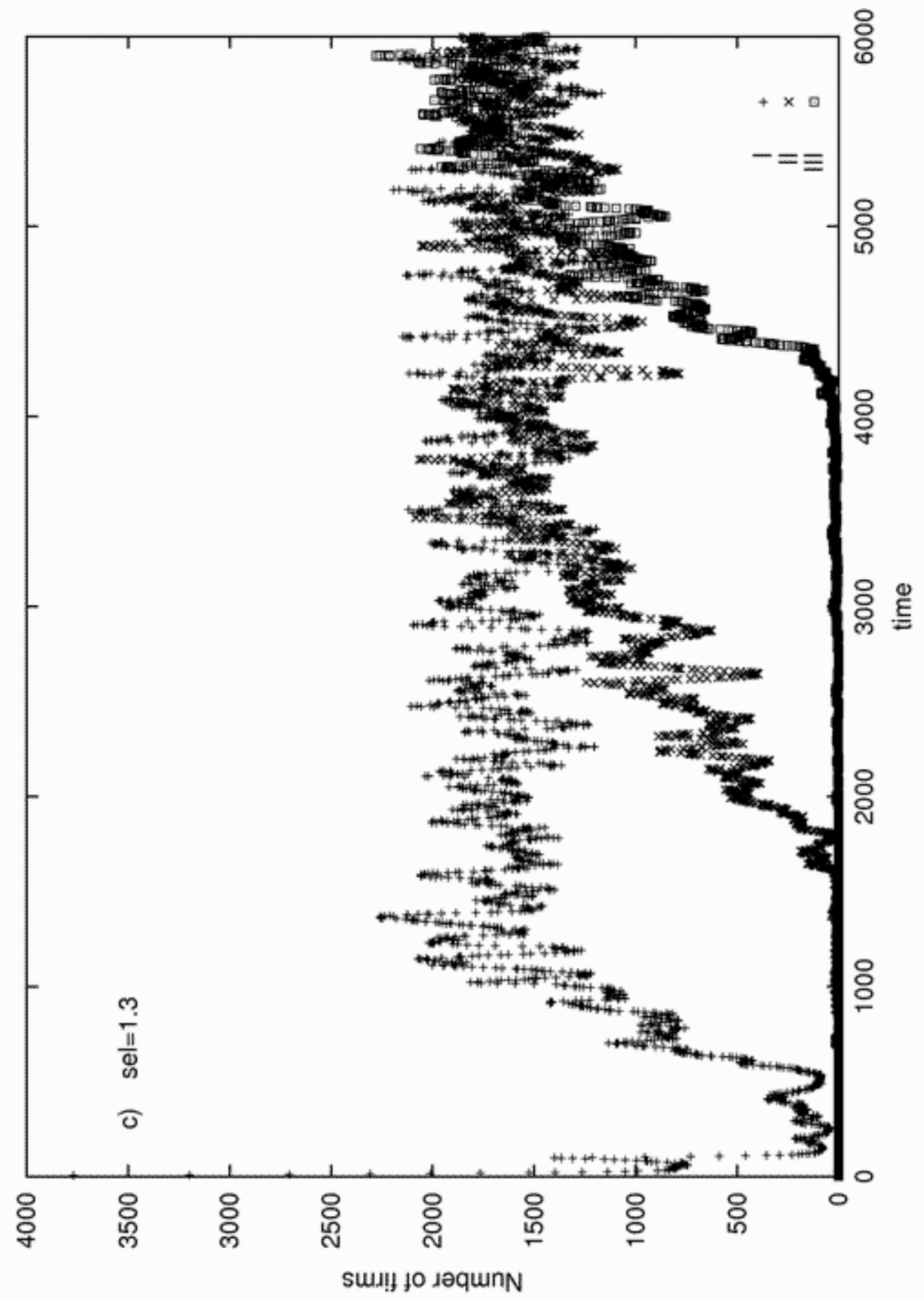




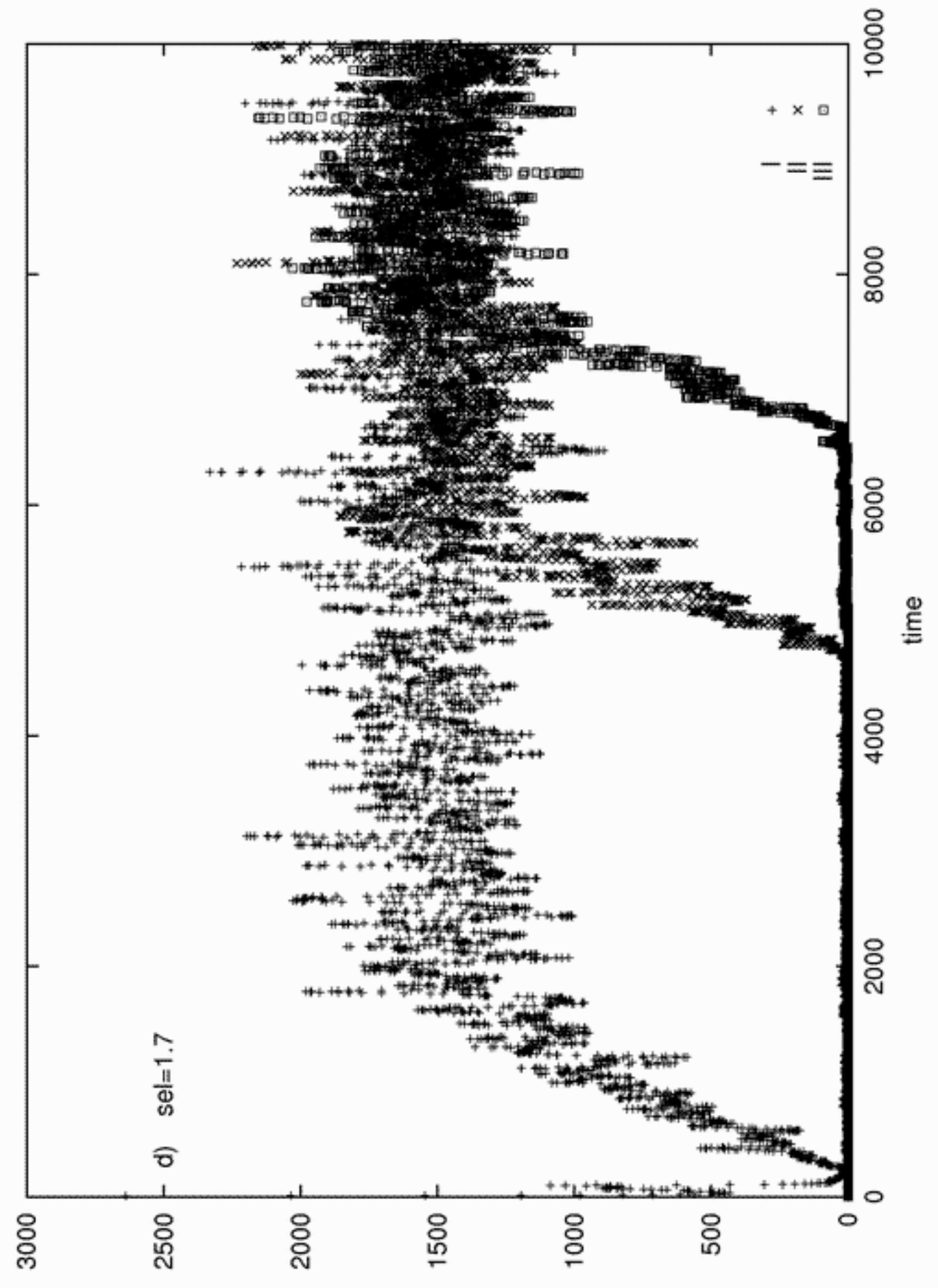

sul! to jəqunN 


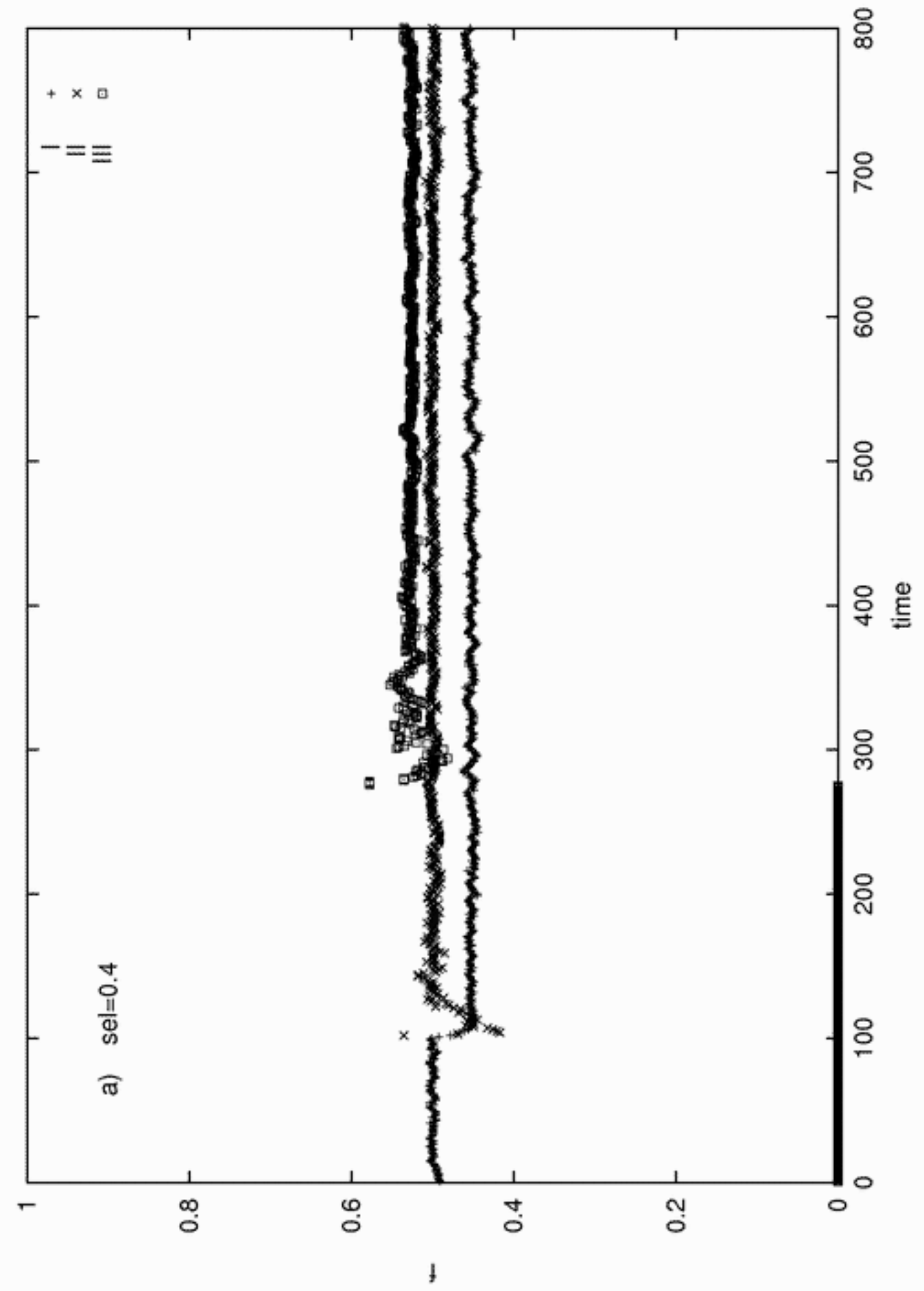




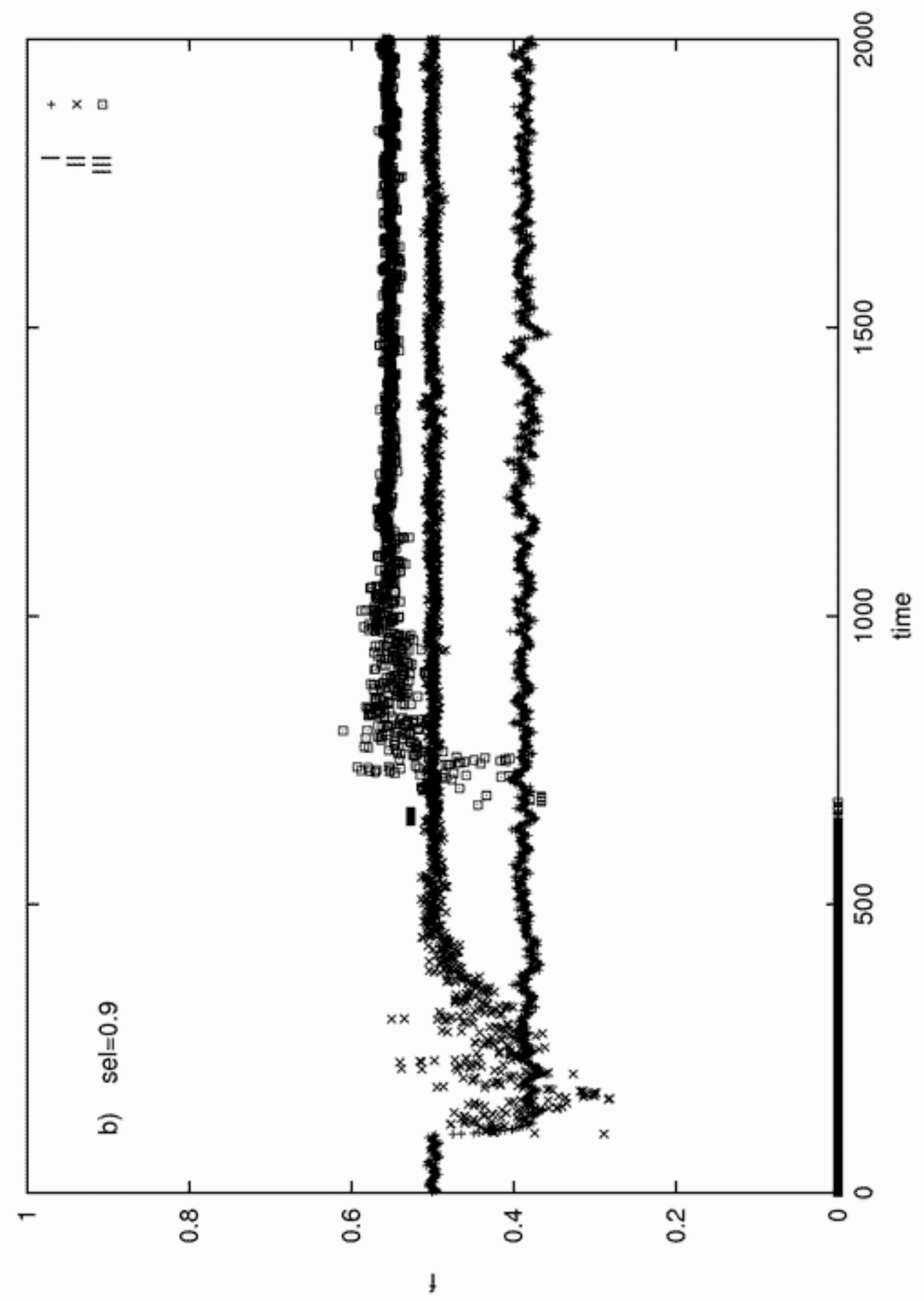




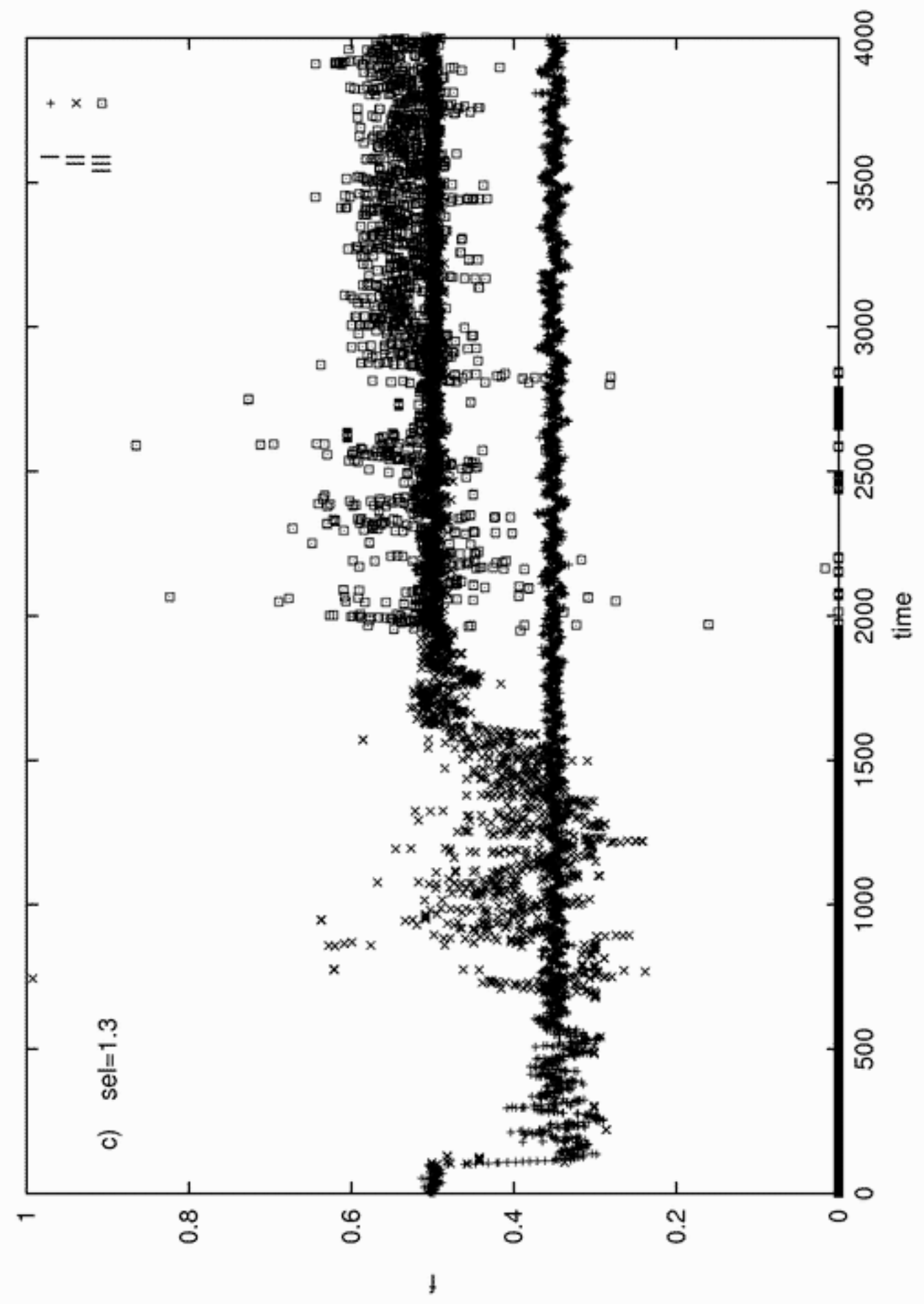




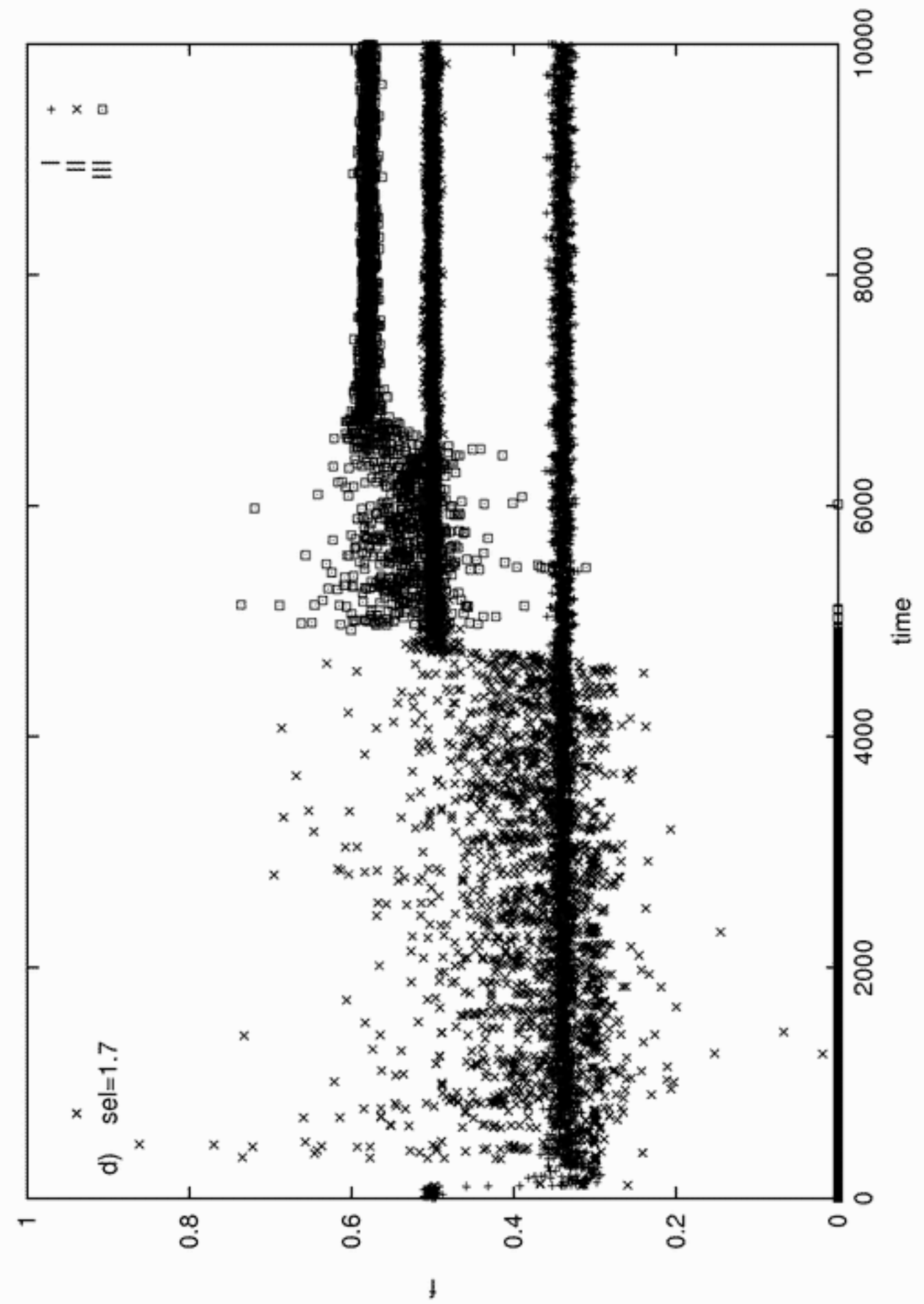




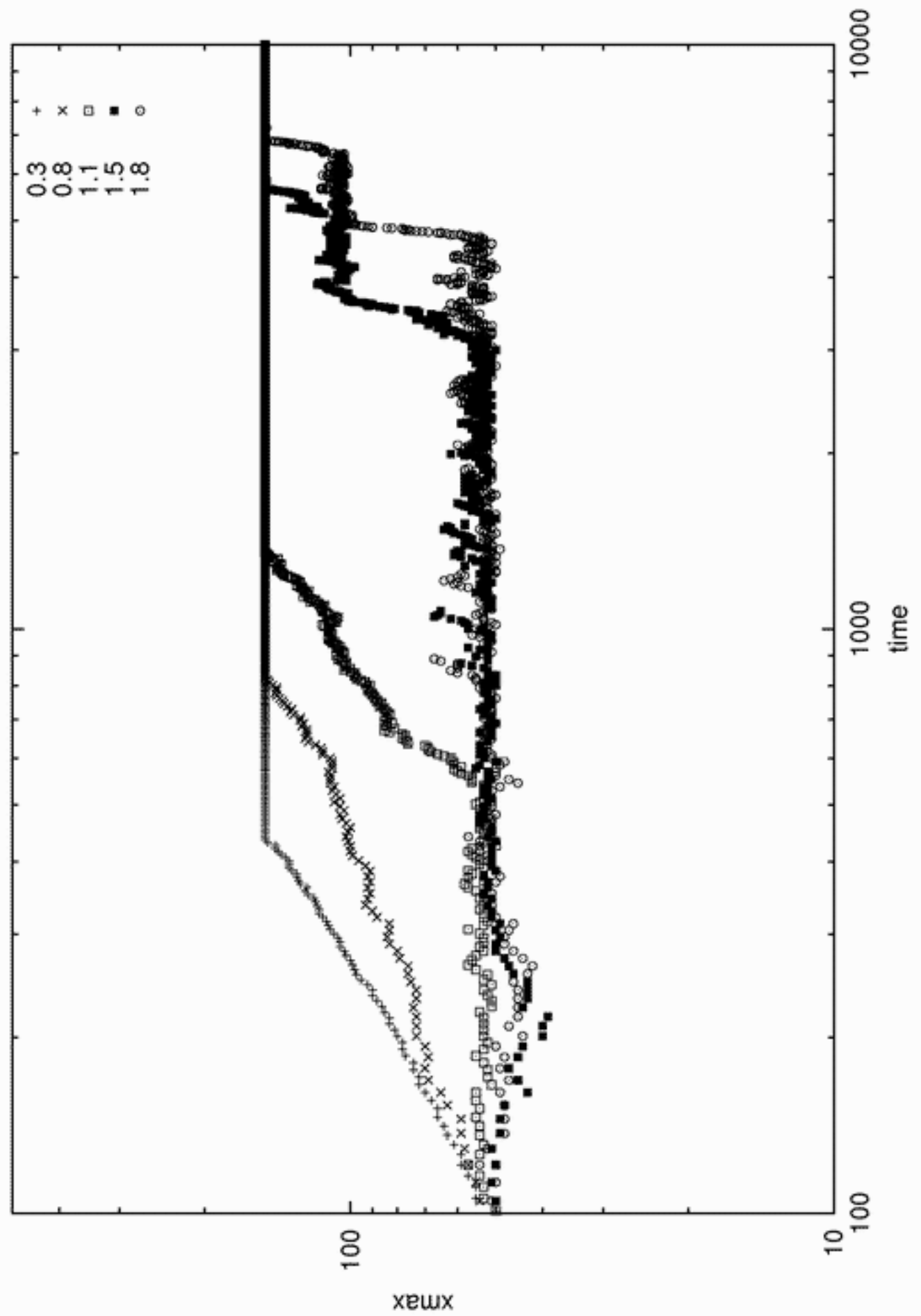




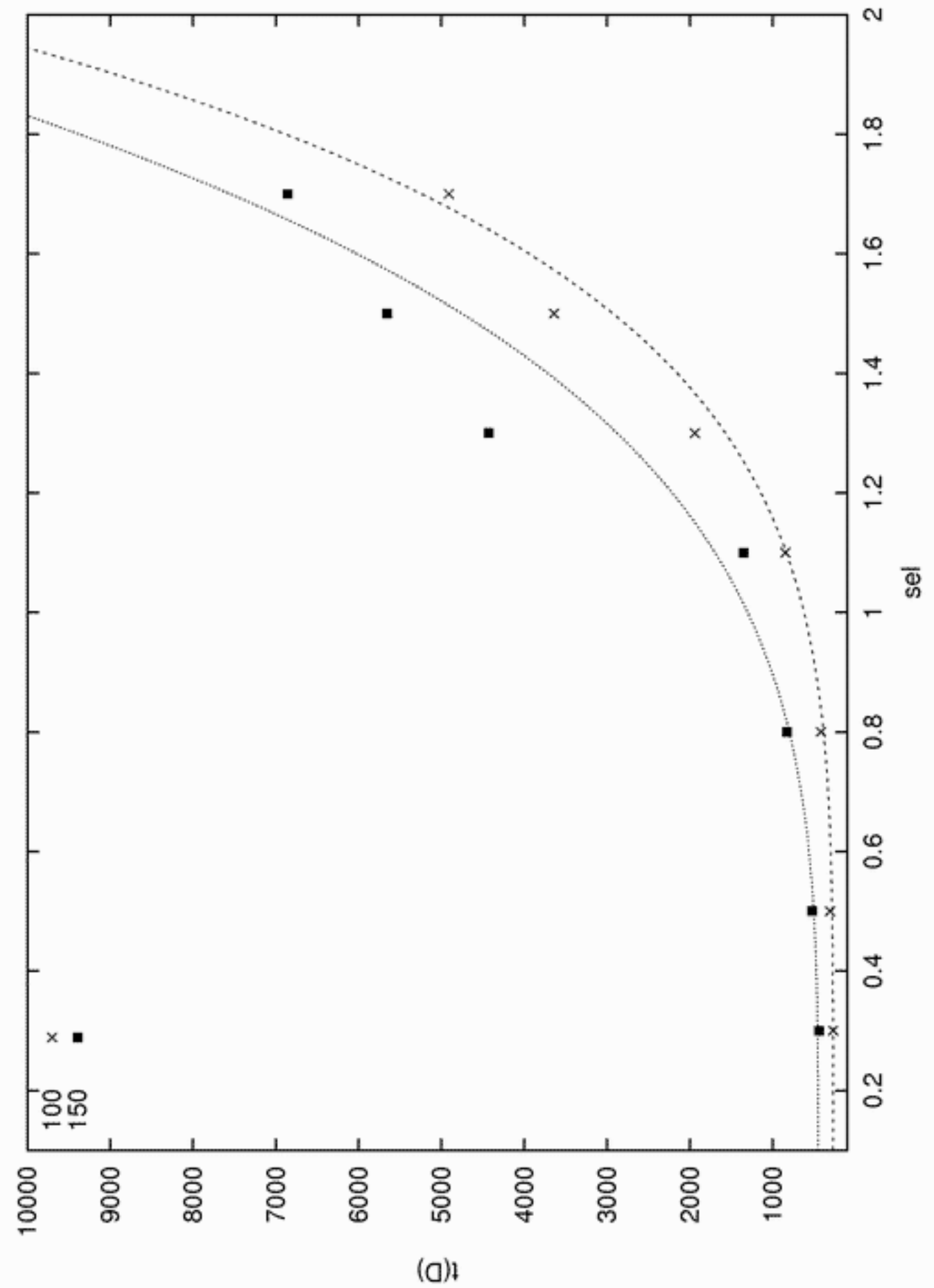




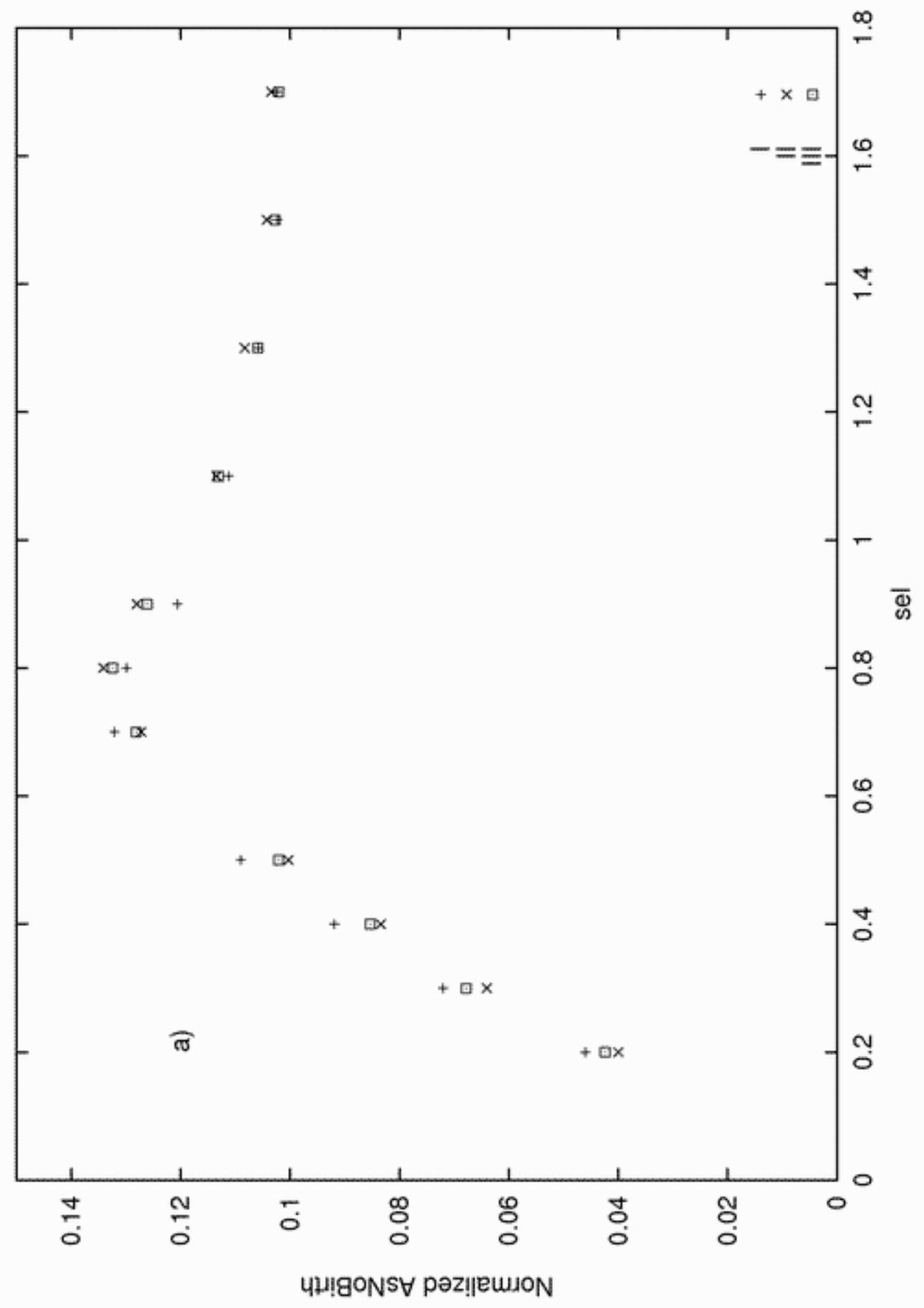




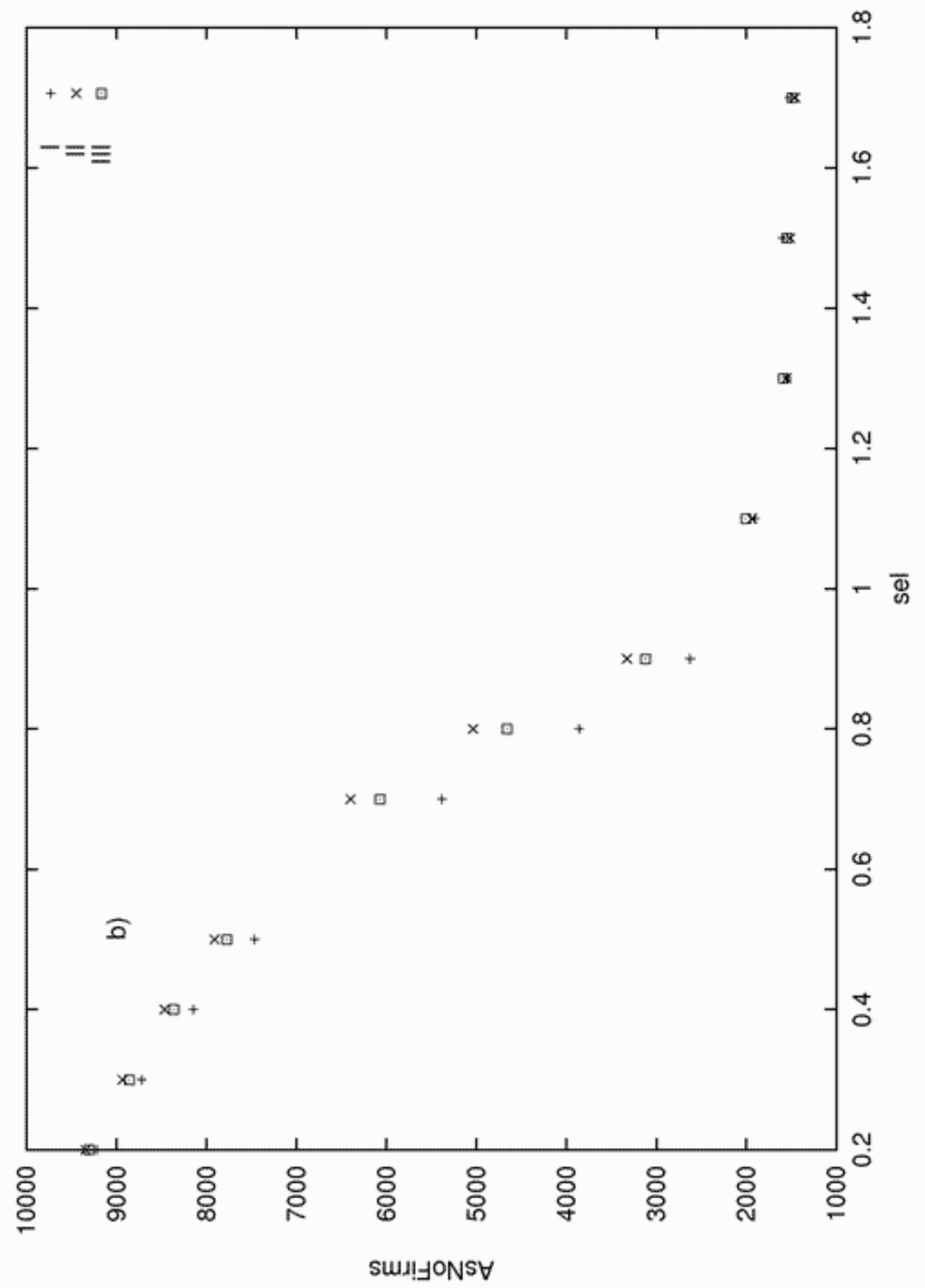




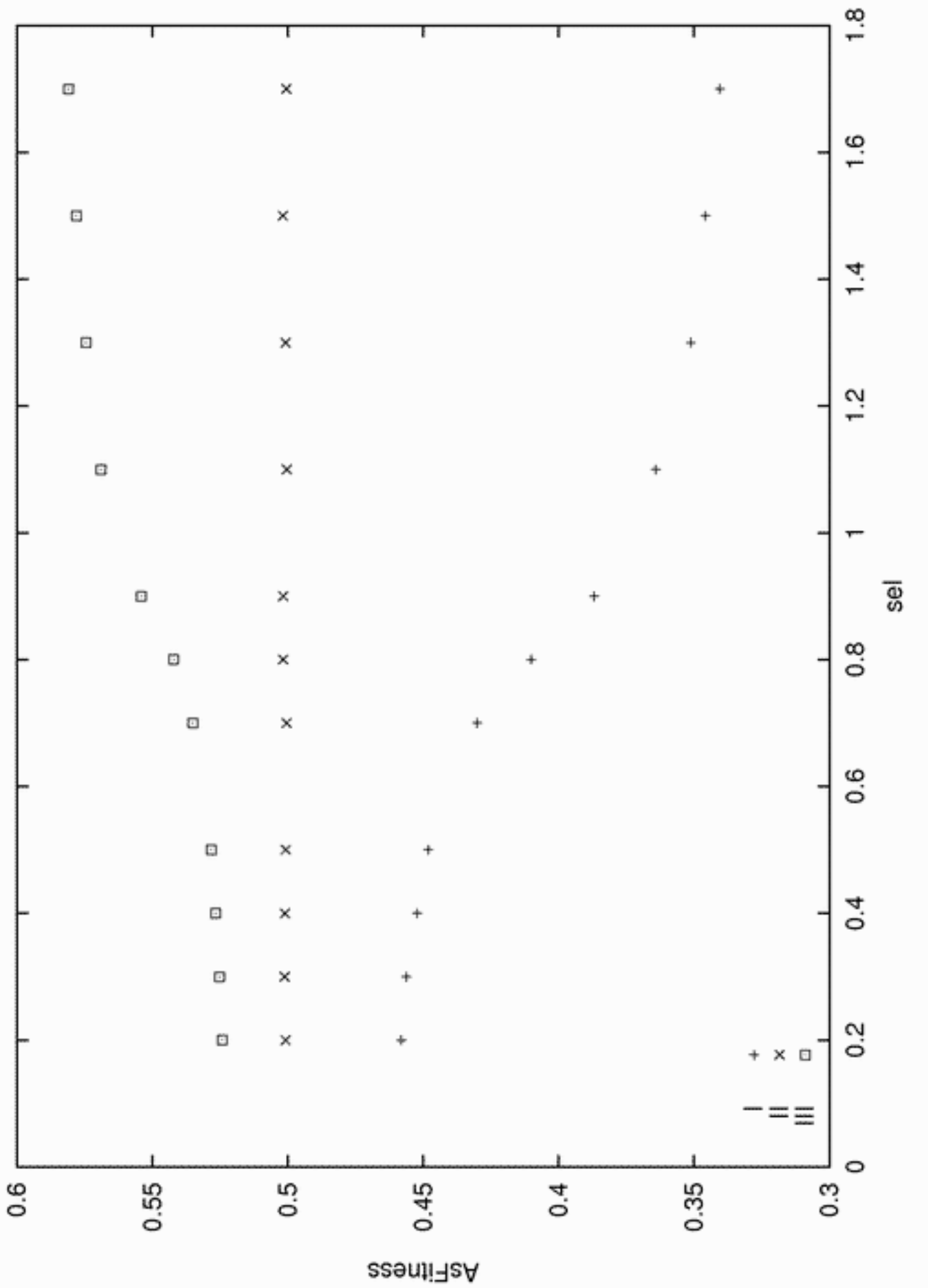




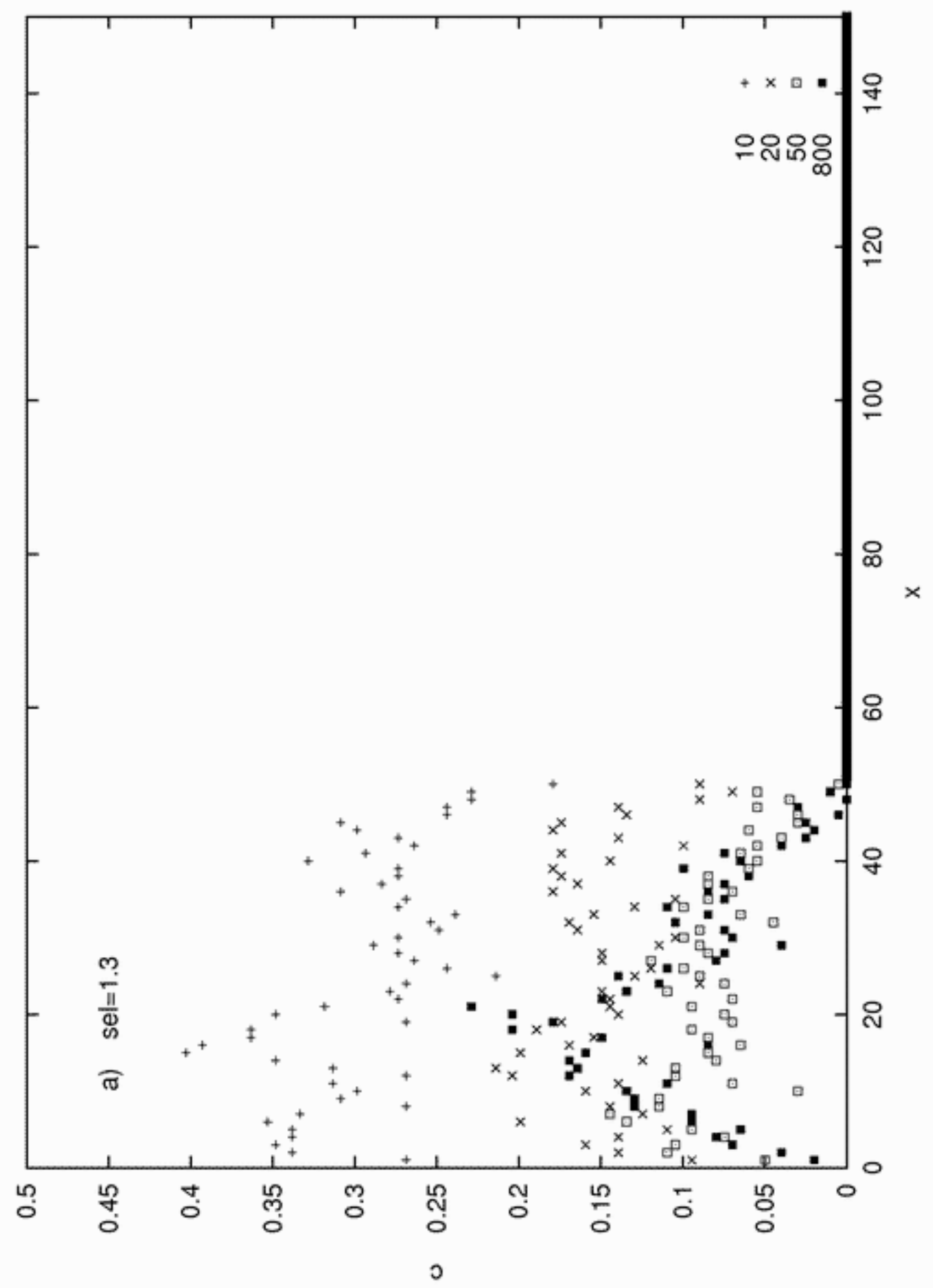




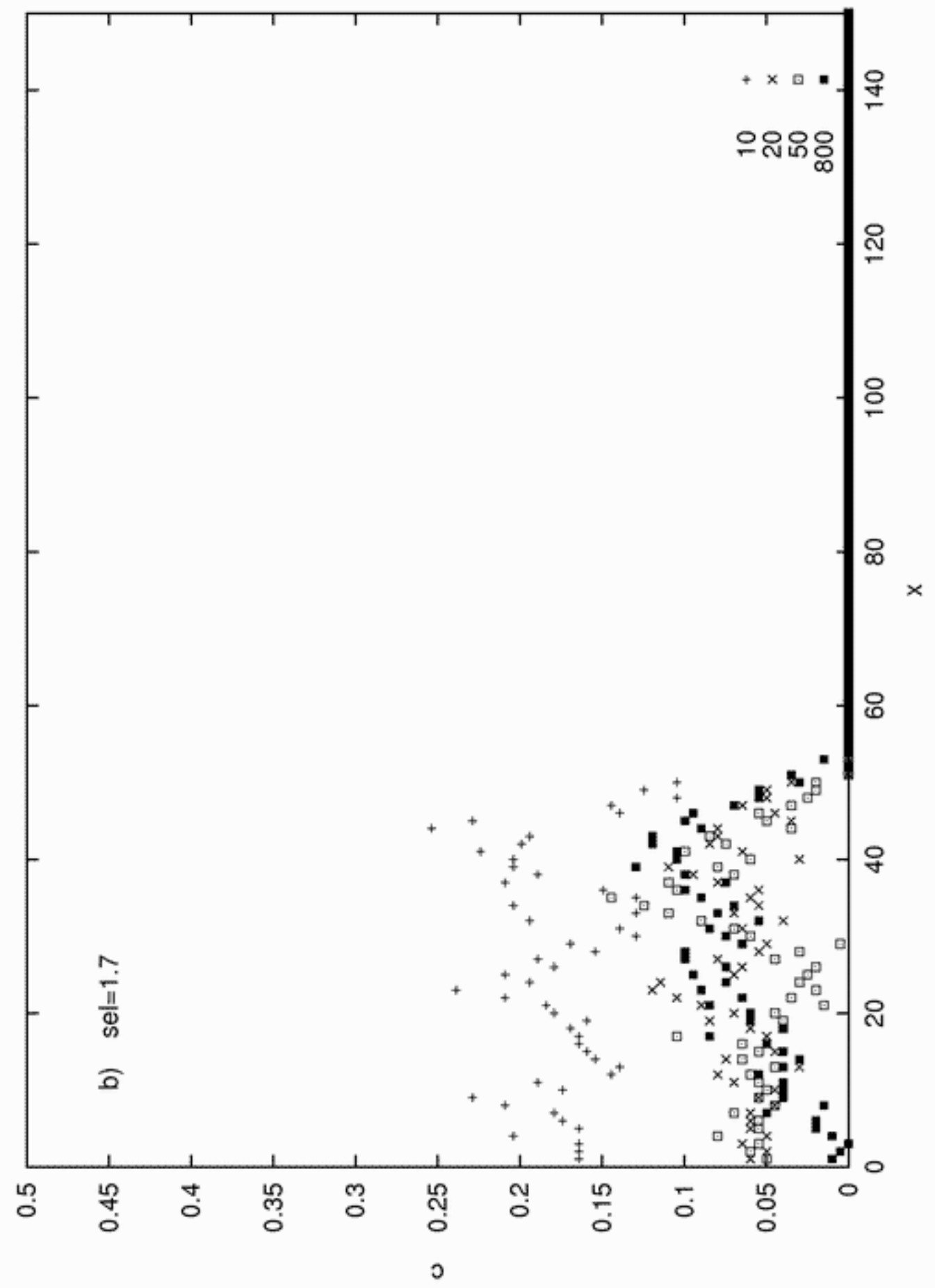




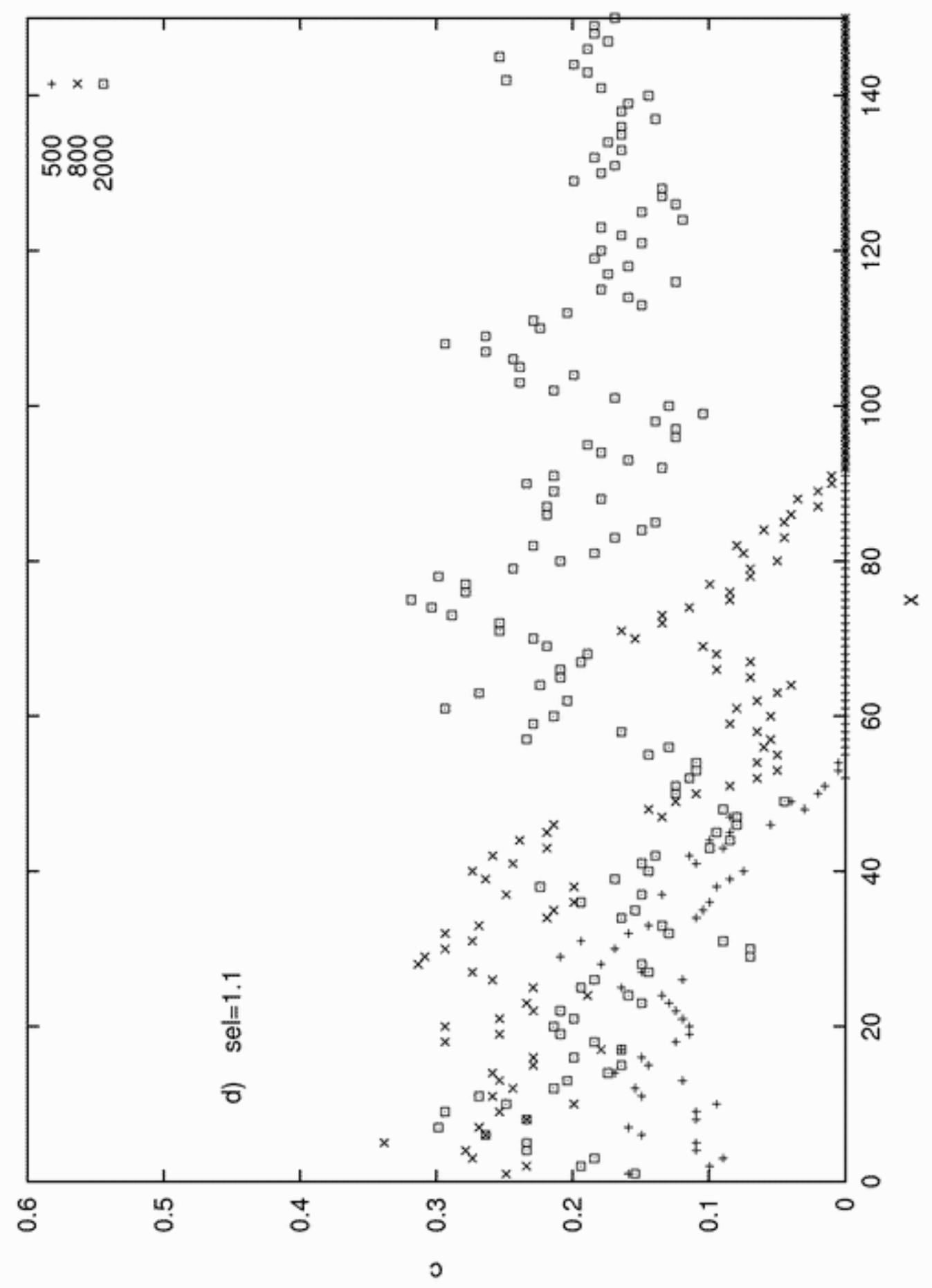




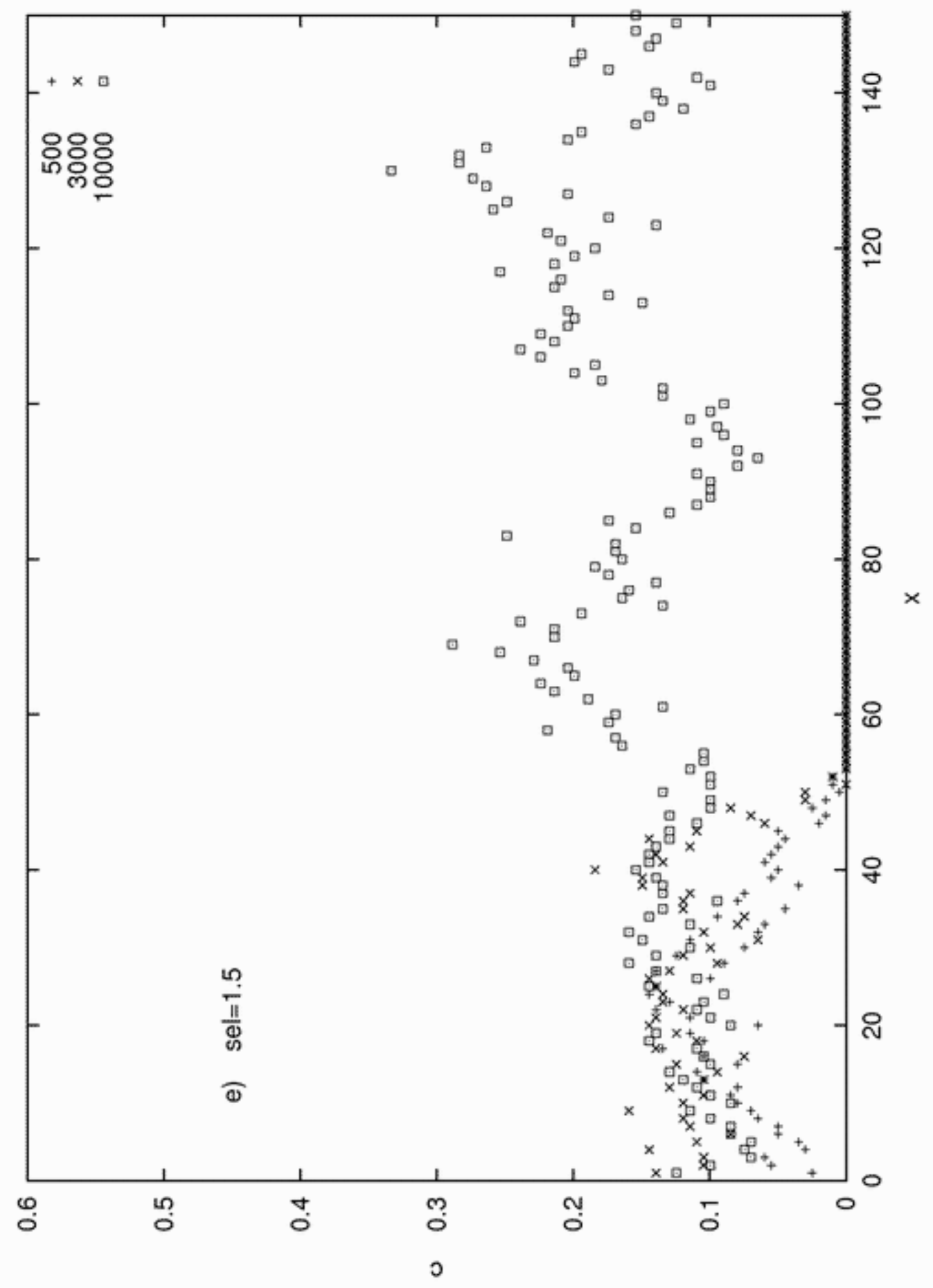




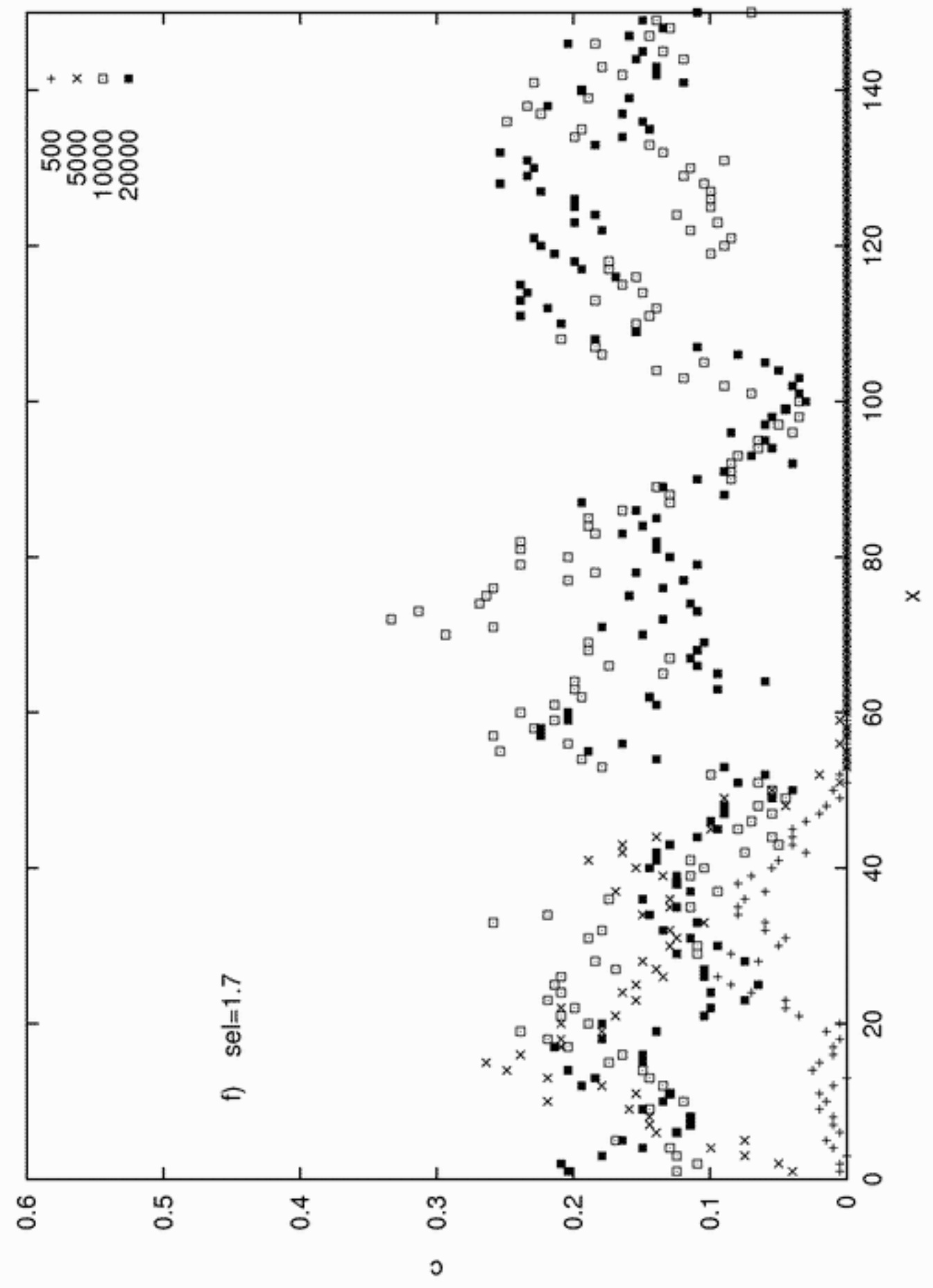




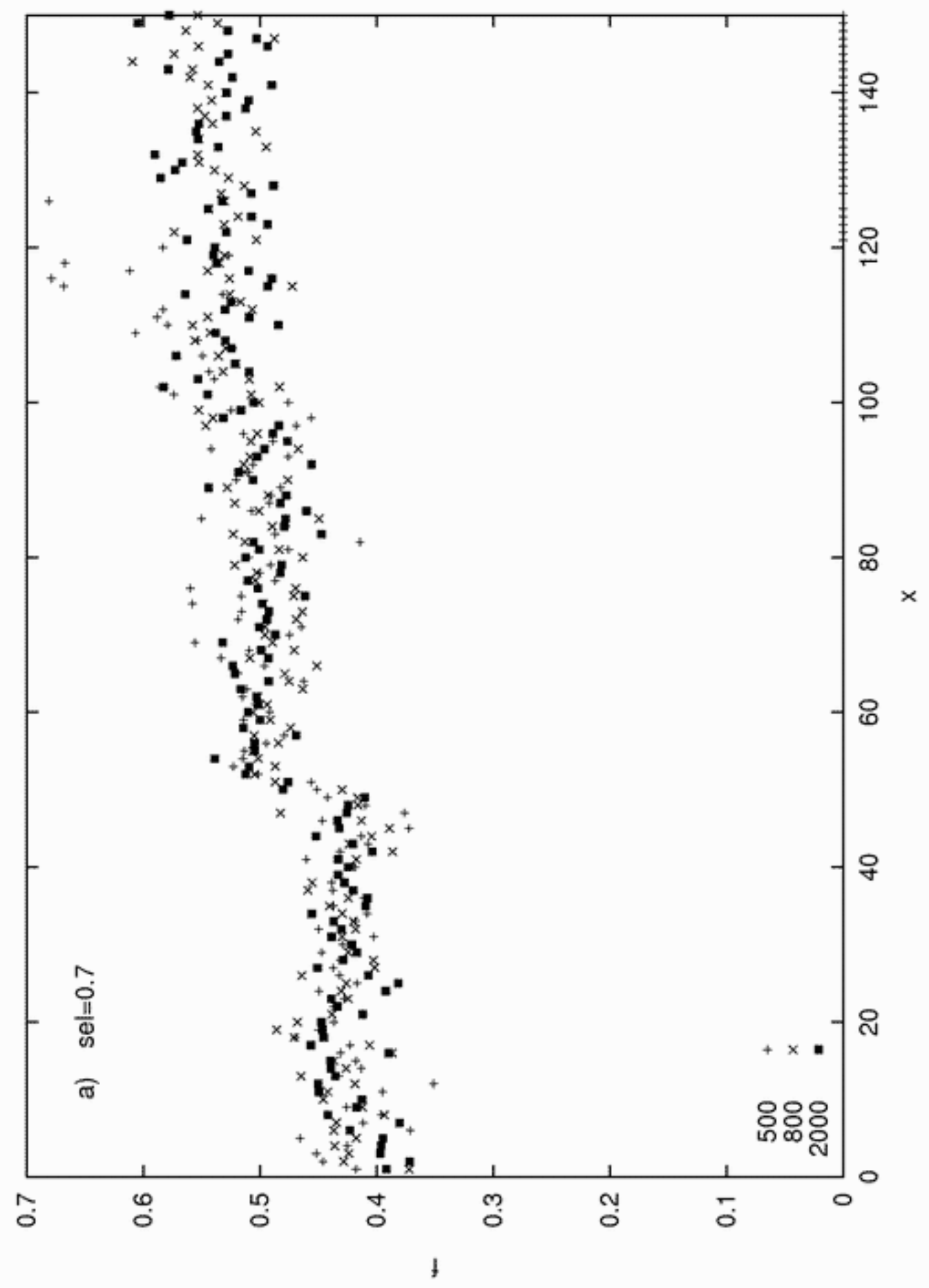




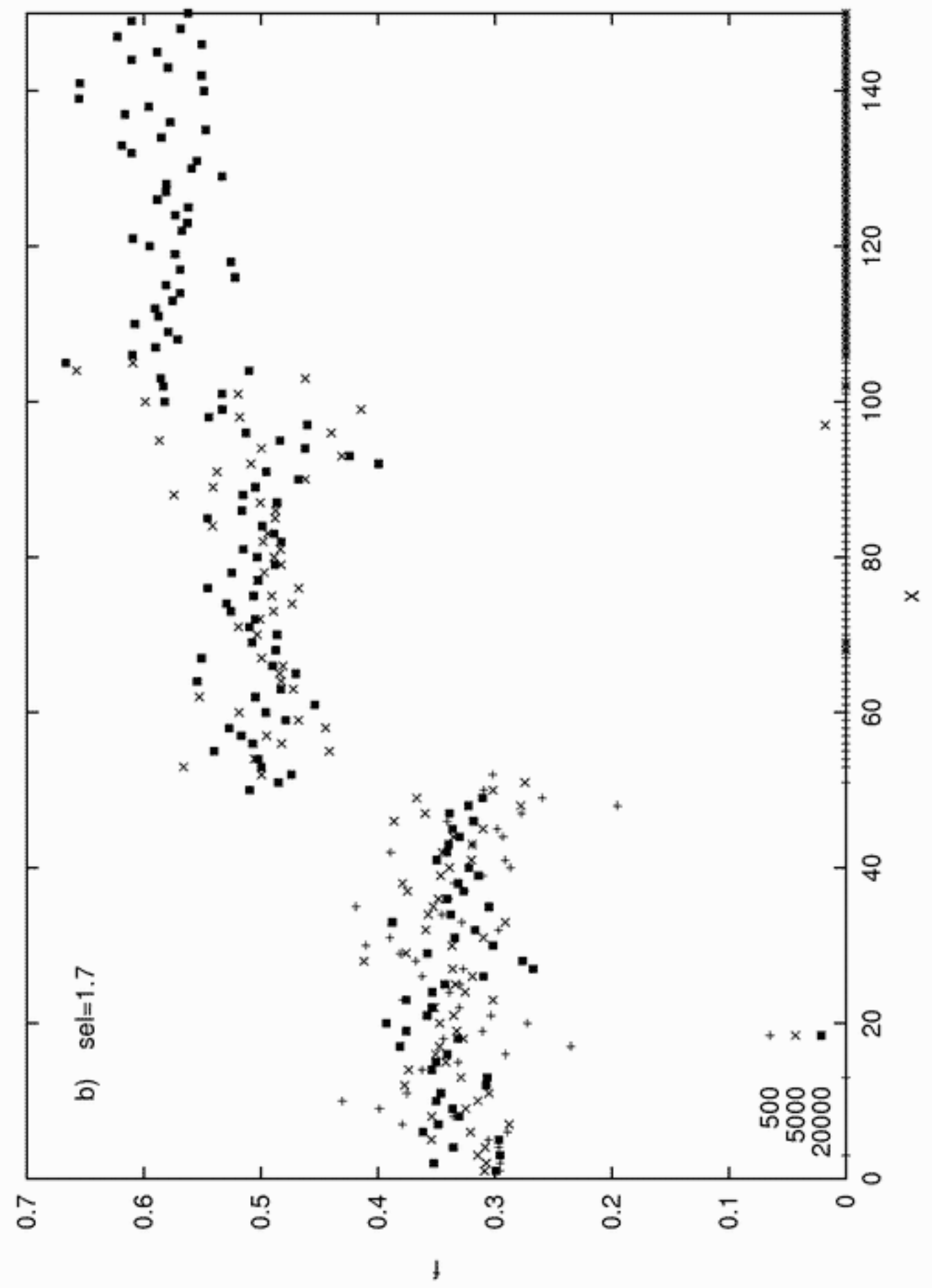

